\title{
Primera década de investigación y educación en la Reserva de la Biosfera Cabo de Hornos: el enfoque biocultural del Parque Etnobotánico Omora
}

\author{
First decade of research and education in the Cape Horn Biosphere \\ Reserve: the biocultural approach of the Omora Ethnobotanical Park
}

Ricardo Rozzi ${ }^{1,2,3}$ \& Elke Schüttler ${ }^{1}$

\section{Resumen}

La Reserva de la Biosfera Cabo de Hornos (RBCH) conserva los bosques más australes del planeta y una de las últimas áreas prístinas que es posible encontrar en el siglo XXI: la ecorregión subantártica de Magallanes en el extremo sur de Sudamérica. A diferencia de la mayoría de las áreas protegidas de Chile, tales como la Reserva de la Biosfera Torres del Paine, que poseen una historia de impactos antropogénicos, la $\mathrm{RBCH}$ ha permanecido protegida por su condición remota y de reserva naval. Hoy, a diez años de la creación de la $\mathrm{RBCH}$, esta condición remota está cambiando y se requiere reforzar un programa de investigación y educación orientado proactivamente hacia la conservación, que contrasta con medidas reactivas de restauración necesarias en otras áreas protegidas. La investigación y educación desarrolladas en el centro científico de la $\mathrm{RBCH}$, el Parque Etnobotánico Omora, ha tenido un enfoque biocultural que integra tres dimensiones: biofísicas, culturales $e$ institucionales. Durante la primera década (2005-2015) de la $\mathrm{RBCH}$, la investigación interdisciplinaria se ha organizado en tres áreas: (1) biodiversidad subantártica poco percibida, (2) investigación ecológica y socio-ecológica a largo plazo, e (3) integración de ética ambiental, educación y conservación biocultural. Con este enfoque biocultural se ha descubierto un "hotspot" mundial de biodiversidad mundial de briófitas, se ha establecido el primer Sitio de Estudios SocioEcológicos a Largo Plazo en la latitud $55^{\circ} \mathrm{S}$, y creado la Filosofía Ambiental de Campo, una metodología de educación que podría constituir un modelo para otros currículos pedagógicos y áreas protegidas.
Las dimensiones biofísicas son bien comprendidas, pero para entender cabalmente las dimensiones culturales e institucionales del enfoque biocultural es indispensable conocer el contexto histórico que ha conducido a establecer el futuro Centro Subantártico Cabo de Hornos el año 2017.

\section{Palabras clave:}

conservación biocultural, ética biocultural, ecorregión subantártica de Magallanes, estudios ecológicos a largo-plazo, filosofía ambiental de campo, historia, metáforas.

\section{Abstract}

The Cape Horn Biosphere Reserve (CHBR) conserves the world's southernmost forests and one of the last pristine areas that can still be found in the 21st century: the sub-Antarctic Magellanic ecoregion. Unlike most protected areas in Chile, such as Torres del Paine Biosphere Reserve, which have a history of anthropogenic impacts, the CHBR has remained protected by its remote location and presence of a naval reserve. Today, ten years after its creation, this remote condition is changing. This requires strengthening the research

\footnotetext{
1 Universidad de Magallanes, Parque Etnobotánico Omora, Puerto Williams, Chile.rrrozzi1@gmail.com $\longrightarrow$

2 Instituto de Ecología y Biodiversidad, Santiago, Chile. eschuttler@gmx.de

3 Sub-Antarctic Biocultural Conservation Program, University of North Texas, Denton, TX, USA.
} 
and education programs for implementing a proactive conservation-oriented approach, which contrasts with the reactive measures of restoration that are necessary in other protected areas today. Research and education developed at the Omora Ethnobotanical Park, the CHBR's scientific center, has had a biocultural approach that integrates three dimensions: biophysical, cultural, and institutional. During the first decade (2005-2015) of the CHBR, interdisciplinary research has been organized into three areas: (1) under-perceived sub-Antarctic biodiversity, (2) ecological and socio-ecological longterm research, and (3) integration of environmental sciences and ethics into biocultural education and conservation. This biocultural approach has been instrumental for discovering a global "hotspot" of global biodiversity of bryophytes, establishing the first site of Long-Term Socio-Ecological Research at latitude $55^{\circ} \mathrm{S}$, and created the field environmental philosophy methodological approach, a pedagogical model that could be adapted in other educational curricula and protected areas. The biophysical dimensions are generally well understood, but to fully understand the cultural and institutional dimensions of this biocultural approach it is essential to better know the historical context that has motivated the creation of the future Cape Horn Sub-Antarctic Center in 2017.

\section{Key words:}

biocultural conservation, biocultural ethics, field environmental philosophy, history, metaphors, socio-ecological research, sub-Antarctic Magellanic ecoregion.

\section{INTRODUCCIÓN}

Hace diez años, en su reunión anual del 27 de junio de 2005, el Consejo Internacional de Coordinación del Programa sobre el Hombre y la Biosfera de la Organización de las Naciones Unidas para la Educación, la Ciencia y la Cultura, UNESCO, aprobó la creación de la Reserva de la Biosfera Cabo de Hornos postulada por el Gobierno de Chile (Rozzi et al. 2006a). Las reservas de biosfera son zonas generalmente extensas, de ecosistemas terrestres o costeros/marinos, o una combinación de los mismos, que albergan una diversidad biológica natural y/o cultural única, cuyo valor haya sido reconocido en un contexto internacional por la UNESCO (Guevara \& Laborde, 2008). En la Región de Magallanes y Antártica Chilena se encuentran dos de las nueve reservas de biosfera chilenas, que contrastan en su historia y estado de conservación actual: Torres del Paine y Cabo de Hornos.

El Parque Nacional Torres del Paine fue creado en 1959 y designado como Reserva de la Biosfera Torres del Paine (RBTP) en 1978. $\mathrm{Su}$ creación obedeció principalmente a sus inigualables bellezas escénicas y abundante vida silvestre, incluyendo megafauna emblemática como el camélido más austral del planeta, el guanaco (Lama guanicoe), el carnívoro terrestre más grande de Chile con distribución más amplia en el continente americano, el puma (Puma concolor), el ciervo nativo más grande de Chile, el huemul (Hippocamelus bisulcus), el ave corredora más rápida de Sudamérica, el ñandú de Darwin (Rhea pennata), y el ave voladora de mayor tamaño en el continente americano y emblema nacional de Chile, el cóndor (Vultur gryphus). El criterio de creación de un parque nacional por sus magníficos paisajes y emblemática vida silvestre es heredado de la tradición de conservación establecida por el primer parque nacional en los Estados Unidos de Norteamérica (EE.UU.) en 1872: el Parque Nacional Yellowstone, que también constituye el primer parque nacional del mundo y el que ha sido considerado como prototipo para los parques nacionales posteriores, incluidos los chilenos (Runte, 1997; Sellars, 1997; Jax \& Rozzi, 2004). Este se ubica en una latitud templada equiparable a Torres del Paine y también presenta grandes mamíferos atractivos, como el oso pardo (Ursus arctos), el ciervo rojo (Cervus elaphus), el alce (Alces alces) y el ave del emblema nacional de EE.UU., el águila calva (Haliaeetus leucocephalus) (Jax, 2001). Al igual que el Parque Nacional Yellowstone, el paisaje de Torres del Paine muestra claros signos de la influencia humana, que ha tenido un impacto especialmente marcado a inicios del siglo XX principalmente por colonos europeos (Dollenz, 1991) y hacia fines del siglo XX e inicios del siglo XXI por un turismo masivo desregulado (Villarroel, 1996; Massardo et al. 2001). Antes de que el gobierno de Chile adquiriera el parque, colonos europeos quemaron zonas de bosques 


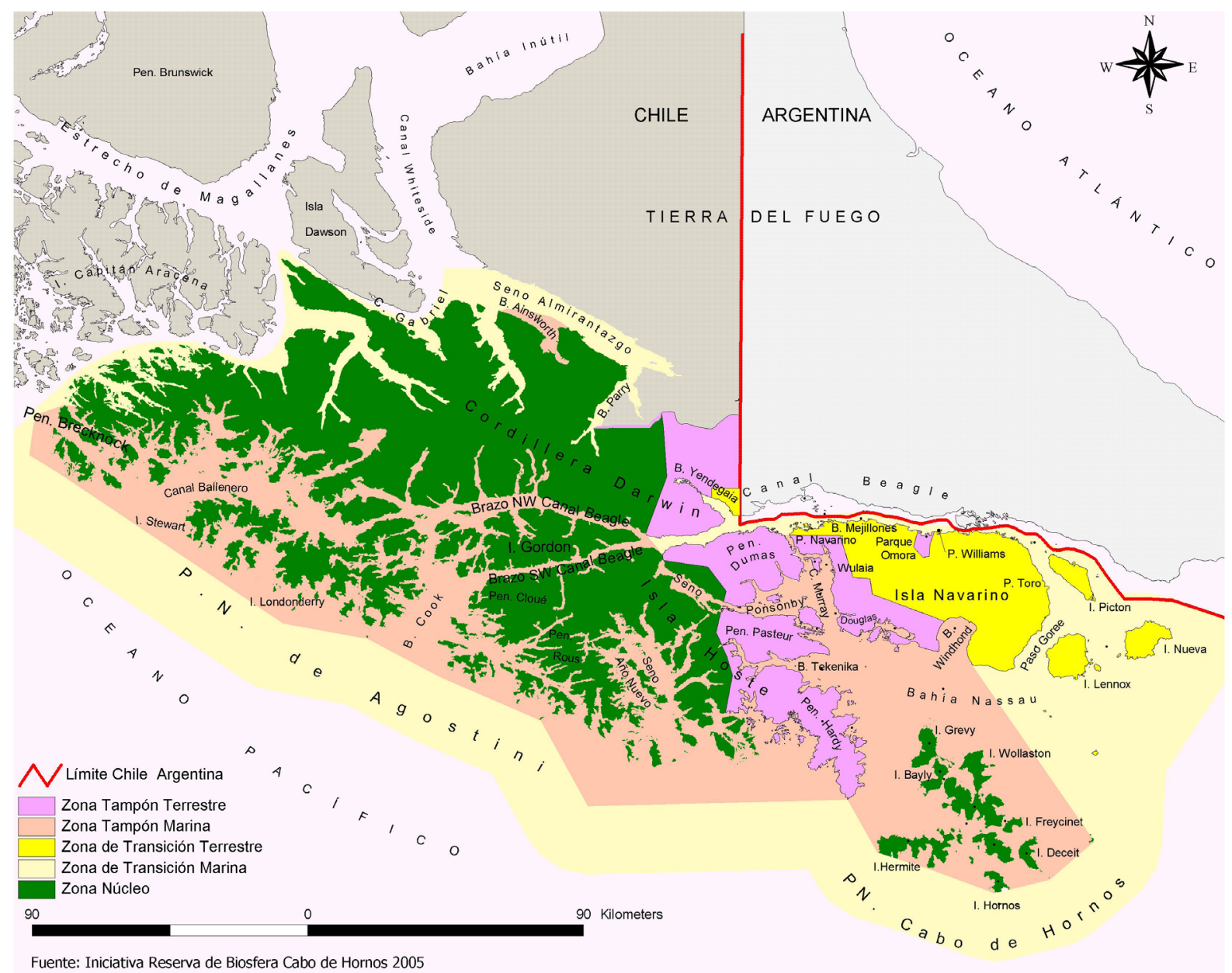

Figura 1. La Reserva de la Biosfera Cabo de Hornos, indicando su zonificación y la ubicación de su centro de investigación y educación, el Parque Omora (zona rosada intensa), en la vecindad de Puerto Williams, capital de la Provincia Antártica Chilena. La zonificación incluye tres tipos de zonas: las zonas núcleo (verde), dedicada a la preservación estricta, que en la actualidad incluye a los parques nacionales de Agostini y Cabo de Hornos, y en el futuro incluirá, total o parcialmente, al Parque Nacional Yendegaia; las zonas de amortiguación terrestre (rosado intenso) y marinas (rosado pálido) que permiten actividades de bajo impacto, tales como la pesca artesanal y el turismo de intereses especiales; las zonas de transición terrestre (amarillo intenso) y marina (amarillo pálido) donde se permiten actividades de mayor impacto, incluyendo la construcción de infraestructura, tales como la futura infraestructura portuaria para cruceros en Puerto Williams y para el cruce en ferry entre Caleta 2 de Mayo en Yendegaia y Puerto Navarino en isla Navarino.

para aumentar la zona de pastos, que fue sobrepastoreada más tarde (Martinic, 1984). En las últimas tres décadas se han generado tres grandes incendios en los años 1985, 2005 y 2011, que han afectado un área de aproximadamente $50 \mathrm{mil}$ hectáreas y han facilitado dramáticos procesos de invasiones biológicas (Vidal \& Reif, 2011).

El turismo en las áreas protegidas conlleva impactos contradictorios y plantea desafíos complejos para la biología de la conservación (Rozzi et al. 2010a). Por un lado, pueden favorecer una mayor integración entre la sociedad y las áreas protegidas, y fomentar el aprecio por aspectos únicos de la biodiversidad como, por ejemplo, las orquídeas de la RBTP, que bajo programas de turismo de intereses especiales bien organizados podrían llegar a ser una flora emblemática (Vidal et al. 2012). Por otra parte, las actuales limitaciones en la planificación y capacidad de regulación del turismo generan impactos ambientales negativos severos en áreas protegidas, como por ejemplo la erosión de senderos, pérdida de calidad de 
visita y provocación de incendios antropogénicos y consecuente introducción de flora exótica en el Parque Nacional Torres del Paine (Villarroel, 1996, Massardo et al. 2001; Vidal \& Reif, 2011).

La actual situación de deterioro causado por el turismo masivo en la RBTP ha demandado un programa de restauración ecológica (Navarro et al. 2015; Repetto \& Cabello, 2015, Vidal et al. 2015). Esta situación contrasta con el estado de conservación de la Reserva de la Biosfera Cabo de Hornos ( $\mathrm{RBCH}$ ) que gracias a su condición remota y la presencia de la Armada de Chile a partir de 1950 ha estado libre de un impacto humano alto sobre la biodiversidad en las zonas archipelágicas ubicadas al sur de Tierra del Fuego (Rozzi et al. 2006b, 2007). Sus archipiélagos forman parte de la ecorregión subantártica de Magallanes que se extiende entre el Golfo de Penas $\left(49^{\circ} \mathrm{S}\right)$ y la isla Hornos $\left(56^{\circ} \mathrm{S}\right.$ ) (Rozzi et al. 2012a). La ecorregión subantártica de Magallanes ha sido identificada como una de las 24 áreas más prístinas del mundo (Mittermeier et al. 2003), alberga los ecosistemas forestales más australes del mundo (Silander 2000) y posee una biodiversidad única generada por cinco fuentes biogeográficas principales (Tabla 1).

La $\mathrm{RBCH}$ representa hoy una oportunidad única para la conservación, la ciencia regional, nacional y mundial, y para un turismo de intereses especiales cuidadosamente planificado. Para su implementación es fundamental establecer una estrecha colaboración entre los servicios públicos, de turismo y las instituciones académicas dedicadas a la investigación científica para fortalecer formas de planificación del turismo de intereses especiales y la conservación en las áreas protegidas que contribuyan a la sustentabilidad ambiental, social y económica. Para lograr una integración entre la conservación biocultural y el desarrollo sustentable de la región, en agosto de 2005 se estructuró un Comité de Gestión presidido por el Gobierno de la Región de Magallanes, el Gobierno de la Provincia Antártica Chilena, la Municipalidad de Cabo de Hornos y la Armada de Chile. Además, se creó un comité científico con base en el Parque Etnobotánico Omora (Parque Omora) en Puerto Williams, que ha tenido la misión de conducir actividades de educación, investigación y conservación en la $\mathrm{RBCH}$ (Fig. 1).
Durante la primera década de existencia de esta reserva de biosfera, el centro científico del Parque Omora logró consolidarse a nivel nacional e internacional, co-gestando la creación de la Red Chilena de Estudios Socio-Ecológicos a Largo Plazo el 2008 (LTSER-Chile, Armesto et al. 2014) y el Programa Internacional de Conservación Biocultural Subantártica. La Red LTSER-Chile es coordinada por el Instituto de Ecología y Biodiversidad (IEB) que agrupa investigadores de las universidades de Magallanes, de Chile, Católica, La Serena y Concepción, y financiada por CONICYT y la Iniciativa Científica Milenio de MIDEPLAN (Armesto et al. 2014). El Programa Internacional de Conservación Biocultural Subantártica es coordinado por la Universidad de Magallanes (UMAG) e IEB en Chile, en colaboración con la Universidad de North Texas en EE.UU. Este programa mantiene una activa red de colaboraciones con otros centros y reservas de biosferas latinoamericanas, como por ejemplo la Estación Charles Darwin en la Reserva de la Biosfera Galápagos (Rozzi et al. 2010a,b).

En este artículo presentamos los marcos teóricos y estructurales bajo los cuales se ha organizado el programa de investigación y educación del Parque Omora durante la primera década (2005-2015) de la RBCH, y concluimos con un análisis histórico de dimensiones culturales $e$ institucionales que han conducido a la definición de las líneas de trabajo para la próxima década (2015-2025) asociadas a la creación del Centro Subantártico Cabo de Hornos. Esperamos que la presentación de la aproximación a la investigación y educación del Parque Omora ofrezca un modelo para otras reservas de biosfera, como Torres del Paine, y que favorezca políticas proactivas de conservación por sobre programas reactivos de restauración ecológica. La conservación de la diversidad biológica y la conservación de la salud humana tienen muchos elementos en común, y como señalan la medicina preventiva y la sabiduría tradicional, en ambos casos "es mejor prevenir que curar".

Ética Biocultural: Un enfoque desde el Sur del mundo

Chile es heterogéneo. Chile es "múltiples 
Tabla 1. A escala de paisaje, la Reserva de la Biosfera Cabo de Hornos (RBCH) ofrece una notable diversidad de comunidades bióticas con una rica biodiversidad que tiene afinidades con cinco regiones biogeográficas contrastantes (basada de Rozzi \& Jiménez, 2014).

\begin{tabular}{|c|c|}
\hline $\begin{array}{l}\text { Patrón de } \\
\text { distribución }\end{array}$ & Ejemplos \\
\hline $\begin{array}{l}\text { Bipolar: regiones } \\
\text { polares, subárticas } \\
\text { y sub-antárticas }\end{array}$ & $\begin{array}{l}\text { Este patrón de distribución de especies congenéricas presentes en las regiones subantártica de Magallanes y } \\
\text { subártica de Alaska es especialmente prevaleciente entre los musgos de la RBCH. Por ejemplo, Tetraplodon } \\
\text { fuegianus, un musgo endémico del extremo sudoeste de Sudamérica, tiene su especie filogenéticamente más } \\
\text { cercana, T. mnioides, en la costa oeste de Norteamérica (Lewis et al. 2014a). Entre las plantas vasculares un } \\
\text { ejemplo es el género Empetrum que tiene sólo dos especies de arbustos en el mundo: E. rubrum, la murtilla, } \\
\text { con frutos rojos restringida al sur de Sudamérica y zonas subantárticas aledañas, y E. nigrum, con frutos } \\
\text { negros restringida a Alaska y otras zonas subárticas (Donoghue, 2011; Popp et al. 2011). La distribución } \\
\text { bipolar de musgos y de plantas vasculares es generada por la dispersión a larga distancia de aves que migran } \\
\text { entre las regiones subantártica y subártica, que están más interconectadas que lo que se pensaba hasta muy } \\
\text { recientemente (Lewis et al. 2014b). }\end{array}$ \\
\hline
\end{tabular}

Bosques tropicales, Las aves y plantas de los bosques costeros siempreverdes de hoja ancha reflejan las conexiones biogeográficas templados y sub- de los bosques de la $\mathrm{RBCH}$ con los bosques tropicales de América del Sur y Central. Entre las especies antárticos de arbóreas, un género neotropical representativo es Drimys, cuyas especies se distribuyen a lo largo de América Sudamérica del Sur y América Central alcanzando el sur de México (Arroyo et al. 1996). El representante más meridional de este género, el canelo (D. winteri) crece en los bosques siempreverdes en la $\mathrm{RBCH}$. El canelo produce frutos carnosos consumidos por el fío-fío (Elaenia albiceps), que se alimentan de ellos antes de su migración cada otoño desde el Cabo de Hornos hasta la selva amazónica (Jiménez et al. en preparación).

Altoandino Las aves y plantas en cojín ofrecen buenos ejemplos de las conexiones que la biota del Cabo de Hornos tiene con la biota de la alta Puna Andina. Por encima de la línea arbórea, en la RBCH es posible observar aves de los géneros Attagis (perdicitas cordilleranas) y Meladonera (yales cordilleranos y australes) que se distribuyen hacia el norte a lo largo del altoandino (Couve \& Vidal, 2003). Las plantas en cojín del género Azorella crecen por encima de la línea arbórea como también cerca de la costa en la $\mathrm{RBCH}$, en cambio en los Andes de Bolivia, Perú, norte de Chile y Argentina estas plantas crecen cerca de los $4.000 \mathrm{~m}$ de altitud (Armesto et al. 1980).

Antártica y sub- Dos ejemplos conspicuos de la estrecha conexión entre las biotas terrestres y marinas de la ecorregión antártica subantártica de Magallanes y la Antártica están representados por los líquenes de color naranja brillante, Caloplaca hookeri y C. sublobata que crecen sobre las rocas de la costa en la RBCH y la Península Antártica (Goffinet et al. 2012), y por los pingüinos antárticos, el pingüino macaroni (Eudyptes chrysolophus ) y el pingüino de barbijo (Pygoscelis antárctica) que se observan ocasionalmente en la $\mathrm{RBCH}$ (Couve \& Vidal, 2003).

Gondwana: Nueva

El ejemplo más representativo es el género Nothofagus, que en la actualidad sólo se encuentra en el Hemisferio

Zelanda, Australia, Sur: en el bioma de los bosques templados del sudoeste de Sudamérica (Chile y Argentina) y Australasia (este y Sudamérica austral sudeste de Australia, Nueva Zelanda, Nueva Guinea y Nueva Caledonia). Árboles fósiles también se conservan en la Antártica. La presencia de este género ofrece un ejemplo vivo de las conexiones gondwánicas que el sur de Sudamérica tuvo con la Antártica, Nueva Zelanda y Australia, hasta finales del Cretácico, hace 75 millones de años (Veblen et al. 1996).

Chiles" en sus realidades biofísicas y culturales. En Cabo de Hornos, en el extremo sur del Continente Americano, hemos establecido un programa de ética biocultural que recupera la comprensión de los vínculos indisolubles entre los hábitos de vida, los hábitats donde éstos tienen lugar, incluidas las interrelaciones con las comunidades de cohabitantes (humanos y otros-que-humanos) (Rozzi et al. 2008a). Esta integración biocultural contrasta con las principales escuelas de la ética moderna (e.g., el utilitarismo y la ética deontológica; Palmer, 2013; Aguirre, 2015) que prevalecen hoy en la sociedad global, y que se centran exclusivamente en los hábitos humanos sin considerar sus hábitats: "como si" los individuos y sus identidades pudieran existir en aislamiento de su medioambiente.

Bajo la ausencia del hábitat en su horizonte conceptual, la ética moderna ha sido esencialmente antropocéntrica; es decir, centrada exclusivamente en hábitos y habitantes humanos. La omisión conceptual del hábitat en la ética moderna también ha facilitado que ésta sea esencialmente eurocéntrica. Esta "ceguera filosófica" promueve programas educativos y políticas de desarrollo uniformes (Rozzi 
et al. 2008a), que con frecuencia provocan grandes pérdidas de diversidad biológica y cultural, que a la vez conllevan injusticias socio-ambientales (Rozzi, 2013). La ética biocultural contrarresta esta "ceguera" mediante la integración de un marco conceptual ecológico-filosófico que afirma el valor vital de los vínculos entre los hábitos de vida, los hábitats y los co-habitantes. Las " $3 \mathrm{Hs}$ " de la ética biocultural involucran, a su vez, tres dominios de la realidad socio-ecosistémica: biofísico, simbólico-lingüístico e institucional-socio-político-tecnológico (Fig. 2).

El programa de investigación y educación del Parque Omora pretende observar, valorar y proteger el extremo austral de Sudamérica desde la macro-escala planetaria hasta la micro-escala de los pequeños seres vivos, las formas de saber $y$ los valores que hoy quedan frecuentemente inadvertidos por la educación, la toma de decisiones y la cultura chilena. Con el marco conceptual de la ética biocultural, este programa procura visibilizar y cuidar:

- un hábitat que carecía de nombre propio, la ecorregión subantártica de Magallanes (Rozzi et al. 2012a,b);

- hábitos de vida animal y vegetal subantárticos que son mucho menos conocidos que los descritos para la región subártica del Hemisferio Norte (Contador et al. 2014);
- co-habitantes que habitan en una región que era considerada pobre en su biodiversidad y hoy es reconocida como un hotspot mundial de briófitas y otros organismos pequeños (Rozzi et al. 2008b).

Programa de investigación transdisciplinaria del Parque Etnobotánico Omora

El programa de investigación del Parque Omora es dirigido por ecólogos y filósofos ambientales y se organiza en tres áreas:

I) Biodiversidad subantártica poco percibida (conocida y valorada),

II) Investigación socio-ecológica y monitoreo a largo plazo,

III) Integración de la ética ambiental y las ciencias ecológicas en la educación y conservación biocultural.

Cada una de estas áreas contempla, a su vez, tres líneas específicas de estudio (Fig. 3). Estas áreas y líneas temáticas se gestaron a partir de experiencias in situ que permitieron identificar problemas y oportunidades para procurar una sustentabilidad socio-ambiental y la conservación biocultural en una región remota y única. En el cuerpo central de este artículo, presentaremos

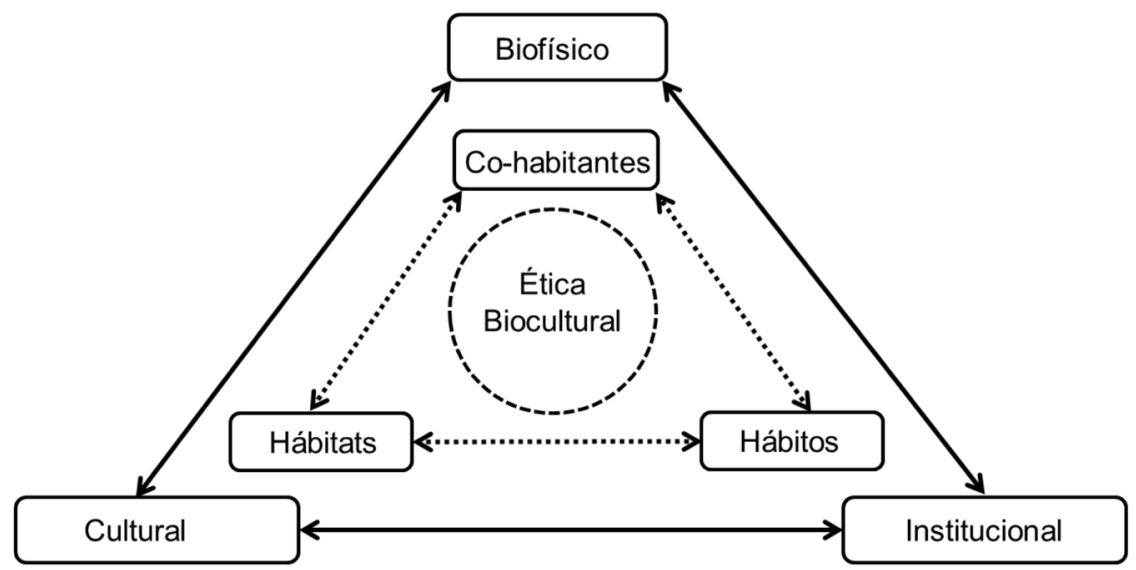

Figura 2. La propuesta formal de la ética biocultural se puede ilustrar mediante una combinación de triángulos incluyentes. Triángulo interior: las " $3 \mathrm{Hs}$ " de la ética biocultural, afirma el valor vital de los vínculos que han co-evolucionado entre hábitos de vida, hábitats y comunidades de co-habitantes. Triángulo exterior: el trabajo en conservación biocultural involucra interrelaciones entre los dominios biofísicos, culturales e institucionales. Las dimensiones biofísicas y culturales de cada región singular, como la Reserva de la Biosfera Cabo de Hornos, deben ser cuidadosamente consideradas por las instituciones, las políticas socio-económicas y la infraestructura. Figura modificada de Rozzi, 2013. 
Tabla 2. Resultados principales de la investigación del Parque Omora en colaboración con instituciones internacionales, nacionales y locales en la Reserva de la Biosfera Cabo de Hornos. Los pictogramas se refieren a las nueve líneas de investigación presentadas en la Fig. 3. Las fuentes corresponden a publicaciones seleccionadas, y el resultado central se destaca en negrita.

\begin{tabular}{|c|c|c|}
\hline \multirow[t]{2}{*}{ Línea } & Resultados principales & Fuentes \\
\hline & $\begin{array}{l}\text { Estudio de la diversidad y ecología de briófitas y líquenes que ha identificado a Cabo de Hornos como } \\
\text { un "hotspot de biodiversidad" de flora no-vascular, y descubierto funciones ecosistémicas esenciales } \\
\text { que las briófitas y líquenes desempeñan en los hábitats terrestres y costeros. }\end{array}$ & 1 \\
\hline \multirow{4}{*}{ W } & $\begin{array}{l}\text { Investigación taxonómica y ecológica de macroalgas que ha caracterizado fenologías con marcada } \\
\text { estacionalidad, identificado especies de alga con valor culinario para el desarrollo de la cocina } \\
\text { regional, y ensayado prácticas de manejo poblacional de macroalgas para su utilización sustentable } \\
\text { por la pesca artesanal. }\end{array}$ & 2 \\
\hline & $\begin{array}{l}\text { Estudios de ecología de macro-invertebrados dulceacuícolas que han descubierto ciclos de vida } \\
\text { multivoltinos de insectos de Cabo de Hornos que difieren de aquellos descritos para taxa similares } \\
\text { en el Hemisferio Norte. }\end{array}$ & 3 \\
\hline & $\begin{array}{l}\text { Programa de Anillamiento y Estudios de Aves de Bosques Subantárticos que ha cumplido } 16 \text { años, } \\
\text { el más prolongado del Hemisferio Sur, y ha detectado marcadas diferencias en patrones migratorios } \\
\text { con bosques subárticos, alto generalismo en la dieta de aves y bajos niveles de endoparasitismo en } \\
\text { paseriformes. }\end{array}$ & 4 \\
\hline & $\begin{array}{l}\text { Investigación sobre plantas y mamíferos exóticos ha incluido la detección y alerta temprana sobre } \\
\text { el impacto del visón sobre la avifauna, especialmente la que nidifica o se alimenta en el suelo y con } \\
\text { uso de hábitat más allá de zonas ribereñas. }\end{array}$ & 5 \\
\hline \multirow[t]{3}{*}{$\underline{\underline{\| N E}}$} & $\begin{array}{l}\text { El equipo del Parque Omora lideró el trabajo técnico-científico de postulación ante UNESCO para la } \\
\text { creación de la RBCH el 2005; luego el Parque Omora asumió la función de centro científico y de } \\
\text { educación en la RBCH, y el } 2015 \text { junto a CONAF ha conformado una unidad ejecutiva de la RBCH. }\end{array}$ & 6 \\
\hline & $\begin{array}{l}\text { Con una filosofía biocultural se ha generado (a) el Programa de Filosofía Ambiental de Campo } \\
\text { que integra las ciencias ecológicas, las artes y la ética ambiental en la conservación biocultural; (b) } \\
\text { una innovación curricular al introducir temáticas de ética ambiental en Chile, y ética biocultural } \\
\text { a nivel mundial; (c) una línea editorial internacional en filosofía ambiental con énfasis en ética } \\
\text { ecológica Latinoamericana, (d) y ha sistematizado las implicaciones éticas del refinado conocimiento } \\
\text { etno-ornitológico yagán y mapuche. }\end{array}$ & 7 \\
\hline & $\begin{array}{l}\text { Programa de educación a múltiples niveles que ha creado (a) el Taller Científico Omora en el Liceo } \\
\text { C-8, Puerto Williams en 2000; (b) el Centro Universitario UMAG Puerto Williams en 2002; (c) el } \\
\text { Programa de Magíster en Ciencias "Manejo y Conservación de Recursos Naturales Subantárticos" } \\
\text { UMAG en 2003, como primer postgrado acreditado de la Patagonia chilena y argentina. }\end{array}$ & 8 \\
\hline & $\begin{array}{l}\text { Conservación biocultural, para su implementación ha generado nuevas temáticas ecoturísticas, tales } \\
\text { como: (a) ecoturismo con lupa (diversidad de líquenes y brioflora subantártica), (b) sumergidos con } \\
\text { lupa (historias de vida de invertebrados dulceacuícolas), (c) ojo bucea con ojo (diversidad y fenología } \\
\text { de invertebrados costeros y macroalgas), (d) etnoecología más austral del mundo (interrelaciones de } \\
\text { la biodiversidad de Cabo de Hornos con la artesanía y conocimiento ecológico tradicional yagán) y } \\
\text { (e) Darwin en el Cabo de Hornos (lugares que inspiraron al joven naturalista a concebir su teoría de } \\
\text { evolución humana). }\end{array}$ & 9 \\
\hline
\end{tabular}

Fuentes: (1) Arróniz-Crespo et al. 2014; Jofre et al. 2010, 2011, Lewis et al. 2014a,b; Méndez, 2013 ; Méndez et al. 2013; Rozzi et al. 2008b; (2) Mansilla et al. 2012, 2013a, 2014a,b; Ojeda et al. 2014, 2015; (3) Contador et al. 2012, 2014; (4) Merino et al. 2008; Reyes et al. 2015; Rozzi \& Jiménez, 2014; (5) Crego et al. 2014, 2015; Ibarra et al. 2009, 2010; Schüttler et al. 2008, 2009, 2010, 2011; Jiménez et al. 2014; (6) Berghöfer et al. 2008, 2010; Jax \& Rozzi, 2004; Rozzi \& Schüttler, 2013; Rozzi, 2004, Rozzi et al. 2006a,b, 2007, 2012a; (7) Aguirre, 2015; Ibarra et al. 2012; Massardo et al. 2012; Poole et al. 2013; Rozzi 2012a,b, 2013; Rozzi et al. 2010b,c, 2014, 2015; (8) Medina, 2013; Pizarro \& Caballero, 2011; Sherriffs et al. 2005; Zárraga et al. 2006; (9) Contador, 2011; Goffinet et al. 2012; Mansilla et al. 2013b; Massardo \& Rozzi, 2006; Ojeda, 2013; Rozzi et al. 2006b, 2010a,d; Rozzi \& Heidinger, 2006. 
la generación de cada área de investigación desde la perspectiva de los tres dominios socioecosistémicos abordados por el Programa de Conservación Biocultural Subantártica del Parque Omora: (a) biofísico, (b) cultural e (c) institucional (Fig. 2), y complementaremos las motivaciones con los resultados principales de la primera década (2005-2015) de investigación y educación en la $\mathrm{RBCH}$ (resumen en Tabla 2).

\section{I) Biodiversidad subantártica poco percibida}

El área de investigación sobre la biodiversidad subantártica poco percibida tuvo su origen en experiencias bioculturales en terreno que mostraban una falta de conocimiento y de valoración de la flora y fauna más diversa y con mayor grado de endemismo en el área de la $\mathrm{RBCH}$.

I.a) En el ámbito biofísico, la región de Magallanes era considerada pobre en biodiversidad debido a que presenta un número bajo de especies de plantas vasculares y de vertebrados terrestres (principalmente mamíferos, reptiles y anfibios) $e$ inferior al de las regiones mediterránea de Chile Central y del bosque templado valdiviano (Armesto et al. 1998; Arroyo et al. 2000). Sin embargo, el trabajo in situ permitió detectar una exuberante diversidad de flora no-vascular (Rozzi et al. 2008b), una fauna singular de invertebrados dulceacuícolas (Contador et al. 2012; Contador et al. 2014) y una abundante cobertura de macroalgas costeras que incluía especies nuevas para la ciencia (Mansilla et al. 2013a).

I.b) En el ámbito cultural, desde el año 2000 se realizaron estudios con escolares y profesores del único liceo de Puerto Williams y con diferentes grupos socio-culturales que habitan en la isla Navarino $(\mathrm{RBCH})$ a quienes se le aplicaron encuestas estructuradas y semi-estructuradas acerca de su conocimiento, valoración y preferencia de especies de flora y fauna (Rozzi, 2001; Arango et al. 2007). Inesperadamente, en la ciudad más austral de Chile embebida en un archipiélago con exuberante flora y fauna nativa, la mayoría de los encuestados mostraron un conocimiento significativamente mayor acerca de flora vascular, fauna de vertebrados y especies exóticas cosmopolitas que no se encuentran en la $\mathrm{RBCH}$, tales como las rosas y los árboles de manzano (Rozzi et al. 2008a). El calafate fue la única especie nativa del Cabo de Hornos incluida entre las plantas más nombradas por los estudiantes. En las encuestas sobre flora, el $91 \%$ de las respuestas incluyó especies originarias de otras regiones de Chile (e.g., alerce) o del planeta (rosas, manzanos, margaritas, eucaliptos, lechugas y naranjos) (Fig. 4). Aún más, el $78 \%$ de las especies de plantas más nombradas por los estudiantes no están presentes en la $\mathrm{RBCH}$; sólo el calafate, las margaritas y lechugas crecen en la isla Navarino. Respecto a la fauna, muy pocos encuestados nombraron invertebrados. De esta manera, la flora y fauna más diversa e idiosincrática de Cabo de Hornos quedaba excluida del conocimiento y el imaginario biocultural de los habitantes de Cabo de Hornos. Sin embargo, los miembros de la Comunidad Indígena Yagán y los residentes antiguos nombraron mayoritariamente especies de plantas y fauna nativa. Por lo tanto, el desconocimiento de la biota es un fenómeno reciente (exacerbado en los últimos 50 años) y que afecta principalmente a la educación formal y a quienes que desde el sector público o privado hoy toman decisiones de desarrollo en la $\mathrm{RBCH}$.

I.c) En el ámbito institucional, se encontró que los programas de estudio y los textos escolares utilizados por los establecimientos de educación pública en Magallanes contienen ejemplos de plantas y animales que incluyen casi exclusivamente flora vascular y fauna de vertebrados. Además, poseen un énfasis en especies de otras regiones, incluyendo rosas y manzanos, y excluyendo casi completamente a los musgos e invertebrados de la región austral (Medina, 2013; Rozzi et al. 2000).

Estos resultados ilustran cómo conceptos universalizantes en la educación formal e informal, como también están presentes en las políticas de desarrollo, con lo que enmascaran la diversidad cultural y biológica local. La rosa y las manzanas tienen una carga cultural en la cultura cristiana occidental (Wolf, 1958; Touw, 1982) y ocupan un lugar central en la economía chilena y mundial actual: las rosas representan más del $66 \%$ de las flores vendidas en todo el mundo y Chile ocupa el quinto lugar entre los exportadores de manzanas en 


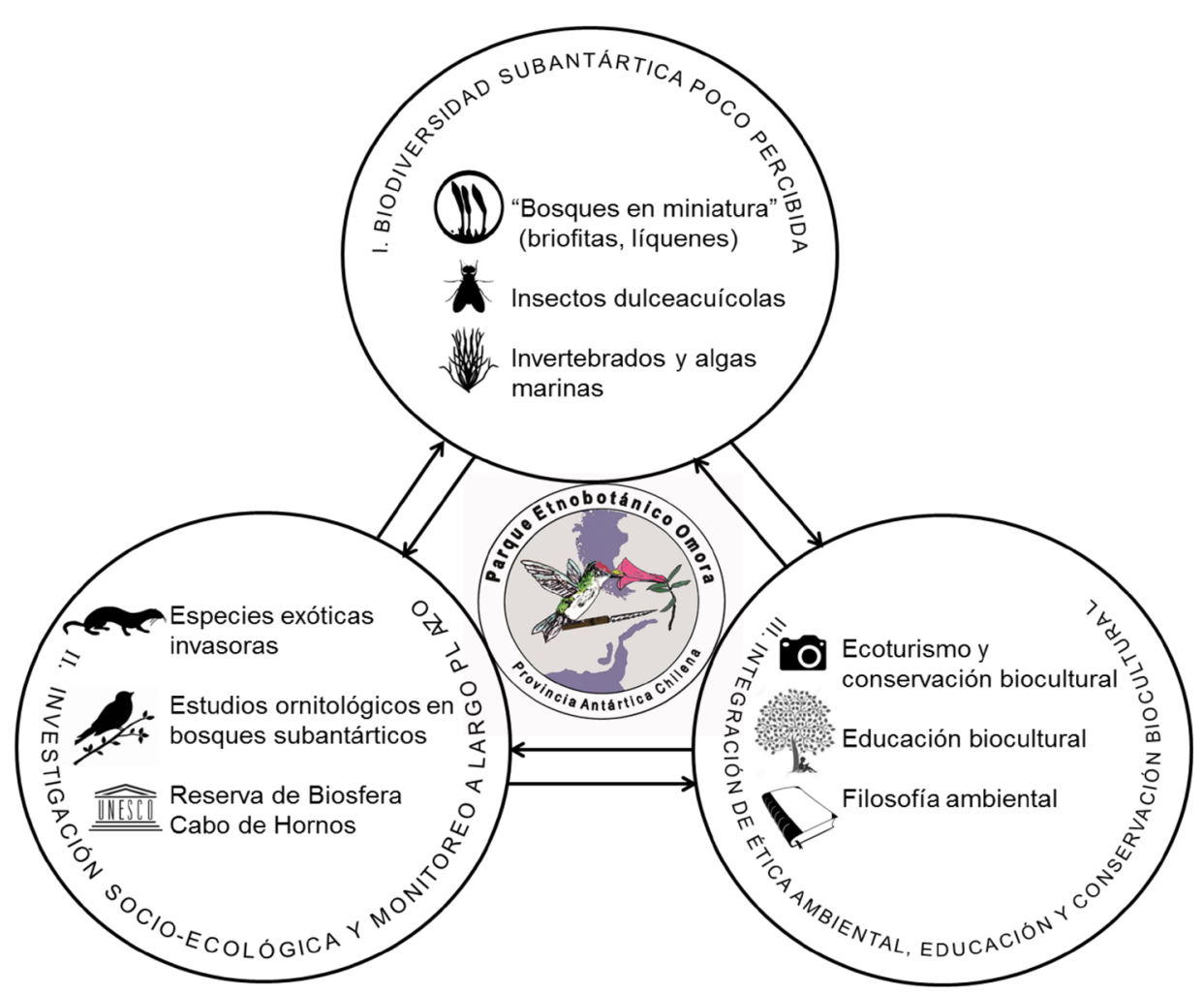

Figura 3. Las tres áreas de investigación del Parque Omora, cada una con tres líneas específicas de estudios.

el mundo (Rozzi et al. 2008a). Por consiguiente, la presencia predominante de las rosas y las manzanas en los imaginarios de los habitantes de Cabo de Hornos, expresaría el lugar central que estas plantas ocupan en la cultura cristiano-europea y el mercado globalizado que hoy alcanza las más altas latitudes australes.

La investigación en el Parque Omora procura reorientar estas tendencias al dirigir sus métodos y temáticas al descubrimiento y enseñanza de la diversidad biocultural local y regional, y utilizar una aproximación biocultural de "filtro fino" que integra distintas formas de conocimiento ecológico sobre grupos de biodiversidad poco percibidos como son la flora no-vascular, los invertebrados dulceacuícolas y las algas marinas (Rozzi et al. 2008a, 2014). En cada uno de estos ámbitos, el trabajo colaborativo con personas e instituciones del ámbito público y privado ha sido efectivo para incorporar a la biota nativa en los programas de educación escolar (Medina, 2013), ecoturismo (Rozzi, 2001; Contador, 2011; Ojeda, 2013, Méndez et al. 2013), y planificación territorial (Rozzi, 2004; Rozzi et al. 2006a). En contraste con el año 2000, hoy los estudiantes del Liceo de Puerto Williams: (a) nombran y reconocen especies de musgos y flora nativa vascular de la $\mathrm{RBCH}$ (Medina, 2013); (b) grupos de estudiantes han ganado premios regionales y en los años 2010 y 2014 el Premio Nacional de Ciencia Escolar EXPLORACONICYT con estudios sobre los invertebrados dulceacuícolas de la $\mathrm{RBCH}$ (Contador et al. 2014); (c) en la cocina regional se han incorporado varios platos preparados en base a especies de macroalgas de la ecorregión subantártica de Magallanes, incorporando las algas en la cocina regional, una práctica que no había sido utilizada en Magallanes, como ocurre en otras regiones de Chile y el planeta (Mansilla et al. 2013b). Estos resultados obtenidos por el Programa Internacional de Conservación Biocultural Subantártica coordinado por la UMAG, IEB y UNT en el Parque Omora durante el período 2000-2015, demuestran que el grado de conocimiento y valoración de la biodiversidad subantártica pueden ser positivamente reorientados para favorecer prácticas de uso sustentable y conservación. 


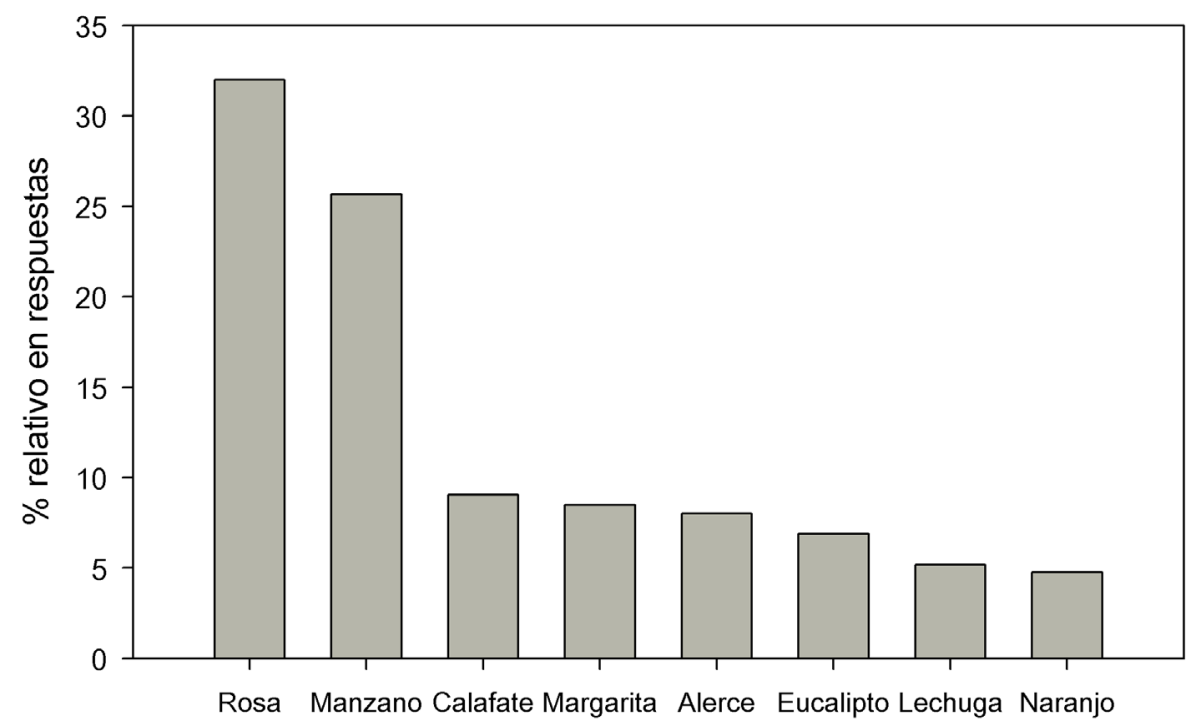

Figura 4. Porcentaje relativo de las especies de plantas más nombradas en las respuestas de alumnos del segundo ciclo de enseñanza básica $\left(5^{\circ}\right.$ a $8^{\circ}$ básico) en el Liceo C-8 de Puerto Williams en marzo del año 2000. En esa fecha se inició el Taller Científico Biocultural Omora como actividad de libre elección en este liceo, y se ha mantenido ininterrumpidamente hasta el 2015 (datos Rozzi, 2001).

II) Investigación socio-ecológica y monitoreo a largo plazo

La investigación socio-ecológica y monitoreo a largo plazo se consolidó cuando en el Parque Omora el año 2008 se formalizó la creación de la Red Chilena de Estudios Socio-Ecológicos a Largo Plazo (LTSER-Chile) que comprende tres sitios cofundadores: Parque Nacional Bosque Fray Jorge (30S; Gutiérrez et al. 2010), Estación Biológica Senda Darwin $\left(42^{\circ}\right.$ S; Carmona et al. 2010) y el Parque Etnobotánico Omora $\left(55^{\circ} \mathrm{S}\right.$; Rozzi et al. 2010b).

II.a) En el ámbito biofísico, esta segunda área de investigación del Parque Omora procura comprender de manera precisa las diferencias biológicas y físicas entre las regiones subpolares de los hemisferios sur y norte. Para ello ha establecido redes de colaboración con sitios de estudios a largo plazo en el subártico, y ha determinado un marcado contraste entre patrones climáticos oceánicos en los bosques más australes y boreales de América en Cabo de Hornos y Fairbanks, Alaska, respectivamente (Fig. 5). Estas diferencias climáticas generan a su vez diferencias bióticas entre las zonas subantárticas y subárticas que incluyen la estacionalidad fenológica de plantas y algas (Ojeda et al. 2015), los ciclos de vida de insectos dulceacuícolas (Contador et al. 2014) y la longevidad y conductas migratorias de las aves (Rozzi \& Jiménez, 2014). Respecto los estudios de aves, el Programa de Estudios Ornitológicos a largo Plazo del Parque Omora fue iniciado en el enero del año 2000, y ha mantenido sin interrupción muestreos mensuales con redes de niebla y anillamiento de paseriformes. Estos estudios ornitológicos del Parque Omora representan hoy el programa de monitoreo continuo de aves con redes de niebla más largo que se realiza en bosques templados y subpolares del Hemisferio Sur (Rozzi \& Jiménez, 2014).

I.b) En el ámbito cultural, hasta ahora a nivel mundial el componente social de los estudios socio-ecológicos se ha centrado en la dimensión económica (Rozzi et al. 2012a). De hecho, la plataforma europea LTSER fue diseñada "[...] como una infraestructura de investigación para apoyar la investigación socioeconómica y ecológica integrada y el monitoreo del desarrollo a largo plazo de la interacción sociedad-naturaleza en el contexto del cambio ambiental global" (Haberl et al. 2009, p. 1798). Esta cita muestra que el término "socio-ecológica" queda a menudo 

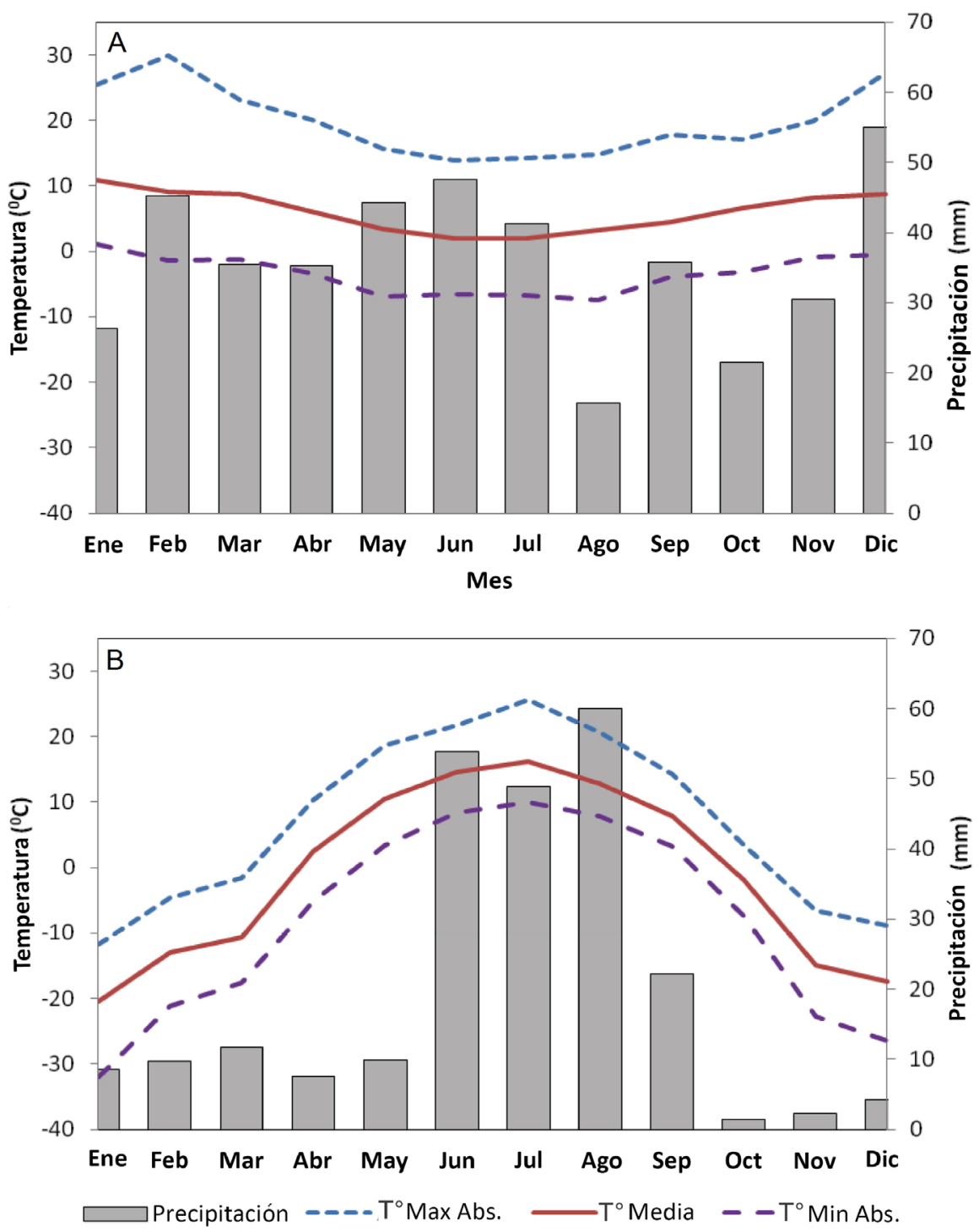

Figura 5. Precipitación media mensual (barras), temperatura media mensual (línea roja central), temperatura máxima absoluta (línea azul discontinua) y temperatura mínima absoluta (línea discontinua púrpura) de la Estación Parque Omora (A, 545'ㅇㅇ's, 67³8'17' 'W), LTSER-Chile, y en la Estación Bonanza Creek LTER-EE.UU. en Alaska) (B, 6442'52”N, 14808'43"W).

Datos climáticos para ambos sitios corresponden al período enero 2009-diciembre 2012.

Fuente: LTSER-Chile (www.ceazamet.cl, 18 de Abril 2015), y LTER-Bonanza Creek (www.lter. uaf.edu, 18 de Abril 2015). Figura modificada de Rozzi \& Jiménez, 2014.

subsumido por "socioeconómico" en documentos fundacionales de las redes LTSER (véase también Parr et al. 2002; Redman et al. 2004, Lui et al. 2007; Ohl et al. 2007; Singh et al. 2007). Frecuentemente la práctica de la ciencia pone poca atención en los contextos sociales y los valores éticos, incluso cuando éstos son fundamentales para la aplicación de la ciencia como es el caso en la biología de la conservación (Chapin, 2004; Heller \& Zavaleta, 2009; Rozzi et al. 2015). Cabe destacar, sin embargo, que más del $99 \%$ de todas las publicaciones generadas en sitios de la Red Internacional de Estudios Ecológicos a Largo Plazo (ILTER) que están indexadas en las 
artes y las humanidades han sido generadas por investigadores que trabajan en sitios ILTER del Hemisferio Sur, en su mayoría asociados a la red LTSER-Chile y más específicamente al Parque Omora (Li et al. 2015). De esta manera el Parque Omora ha contribuido a llenar un importante vacío conceptual y cultural en los programas mundiales de investigación a largo plazo socio-ecológicos, mediante el establecimiento de una base teórica y metodológica para integrar las ciencias ecológicas y la ética ambiental en la teoría y la práctica de los estudios LTSER en Chile y la red internacional ILTER (Li et al. 2015; Rozzi et al. 2012a, 2015).

II.c) En el ámbito institucional, el Parque Omora y la Red LTSER-Chile han contribuido también a llenar un vacío geográfico en la cobertura latitudinal y de biomas en los estudios ecológicos a largo plazo. Por ejemplo, en el año 2010 la Red ILTER incluía 543 sitios, de los cuales 509 (93,7\%) se ubican en el hemisferio Norte, mientras que sólo 34 sitios (6,3\%) estaban en el hemisferio Sur (Rozzi et al. 2012a). Hasta el año 2010 hubo un vacío geográfico absoluto en el ámbito latitudinal $40^{\circ} \mathrm{S}$ - $60^{\circ} \mathrm{S}$ en la red mundial ILTER. Estas latitudes comprenden al bioma de los bosques templados y subantárticos de Sudamérica. Hoy la creación de la red LTSER-Chile y la inclusión de esta red y sus sitios Estación Biológica Senda Darwin $\left(42^{\circ} \mathrm{S}\right)$ y Parque Etnobotánico Omora $\left(55^{\circ} \mathrm{S}\right)$ en la red ILTER han permitido subsanar este vacío geográfico en el monitoreo del cambio ambiental global. Además, la investigación a largo plazo en este bioma no solamente complementa la ciencia predominante en los biomas del hemisferio Norte, sino que también permite comparaciones entre ecosistemas subpolares de Norte- y Sudamérica (Armesto et al. 2014).

El escenario de asimetría inter-hemisférica en los esfuerzos de estudios ecológicos a largo plazo es problemático, puesto que se extrapolan resultados obtenidos en el Hemisferio Norte sobre la región latitudinal equivalente del Hemisferio Sur. Extrapolaciones de este tipo son frecuentes en los modelos de desarrollo impulsados por megaproyectos globales que enmascaran diferencias inter-hemisféricas significativas. El Parque Omora contribuye a subsanar estos sesgos a distintas escalas: a escala regional ha establecido un
Centro de Investigación y Educación de la $\mathrm{RBCH}$, a escala nacional ha inaugurado un sitio co-fundador de la Red LTSER-Chile, y a nivel internacional es la sede del Programa Internacional de Conservación Biocultural Subantártica y constituye el sitio más austral de la Red ILTER en América.

III) Integración de la ética ambiental y las ciencias ecológicas en la educación y conservación biocultural

La integración de la ética ambiental y las ciencias ecológicas tiene uno de sus fundamentos conceptuales en el sentido originario del término "ética", que proviene de la palabra griega "ethos" con un doble significado de "habitar" y "hábitat". Descriptivamente, la forma de habitar un hábitat define el ethos o identidad de los habitantes animales y humanos. Normativamente, demanda el respeto por el vínculo entre ambos y por la comunidad de co-habitantes (humanos y nohumanos) que comparten el hábitat y participan en los hábitos de vida (Rozzi et al. 2008a). La ética ambiental aplicada en el Parque Etnobótanico Omora reintegra en la educación este significado ancestral y ensaya una visión de los habitantes embebidos en sus hábitats y hábitos en el sur de Sudamérica. Esta unidad ética, que a la vez es ecosistémica, recupera la integración entre los ámbitos biofísicos y culturales.

III.a) En el ámbito biofísico, el énfasis está puesto en las singularidades del hábitat subantártico, los hábitos de vida de las especies de plantas y las abundancias y patrones de distribución de las comunidades de co-habitantes vegetales. Este énfasis contribuyó a que a través del programa de investigación a largo-plazo del Parque Omora descubriéramos que en la ecorregión subantártica de Magallanes (menos del 0,01\% de la superficie terrestre del planeta) se encuentra más del $5 \%$ de las especies de briófitas (musgos y hepáticas) descritas a nivel mundial (Rozzi et al. 2008b; Goffinet et al. 2012). Este descubrimiento de un "hotspot" o centro mundial de la diversidad de briófitas estimuló un "cambio de lentes" para observar la biodiversidad en el extremo austral de América.

III.b) En el ámbito cultural, el "cambio de 
lentes" tuvo implicancias no sólo para investigar sino también para conservar la biodiversidad (Rozzi et al. 2008b). La alta diversidad de briófitas subantárticas constituyó uno de los argumentos más sólidos para que la UNESCO nominara la Reserva de Biosfera Cabo de Hornos en el año 2005. Esta es la reserva de biosfera más extensa del Cono Sur de América, y su creación constituyó una novedad a nivel mundial: es la primera vez que en Chile y en el mundo se designa un área protegida en base a la diversidad de musgos y hepáticas. Estos pequeños organismos han sido poco percibidos y valorados no sólo en la Región de Magallanes sino también en la conservación internacional. El "cambio de lentes" para investigar y conservar la biodiversidad conduce de esta manera a un cambio de conciencia y actitud de coexistencia con la biodiversidad subantártica.

III.c) En el ámbito institucional, el Parque Omora ha generado un enfoque socio-ecológico que integra el estudio de las ciencias ecológicas y de la filosofía y la ética, y para incorporar estas disciplinas en programas LTSER ha establecido una nueva metodología: la Filosofía Ambiental de Campo (FILAC). La FILAC proporciona orientación a estudiantes de posgrado y otros participantes para llevar a cabo investigaciones que incluyan a la diversidad biológica menos percibida y valorada (e.g., las briófitas), como también a tradiciones culturales y conceptos filosóficos que han sido desatendidos en la educación formal (e.g., formas de conocimiento ecológico amerindio y valores éticos concebidos por tradiciones de pensamiento occidental que son marginadas de la historia, Rozzi et al. 2008b; Rozzi et al. 2015). La FILAC ha elaborado un modelo pedagógico para la educación que ha sido adoptado (1) para el desarrollo de tesis de pre y posgrado en la UMAG y otras universidades de América Latina (Aguirre 2015), y (2) en la enseñanza básica y media del Liceo C-8 de Puerto Williams con involucración de investigadores y estudiantes de postgrado de la UMAG.

El concepto de la FILAC comprende un ciclo de cuatro pasos. El primer paso es la investigación ecológica y filosófica. El segundo paso es la composición de metáforas tales como "bosques en miniatura del Cabo de Hornos". Esta metáfora facilita la comprensión de que las diversas comunidades de musgos, hepáticas,

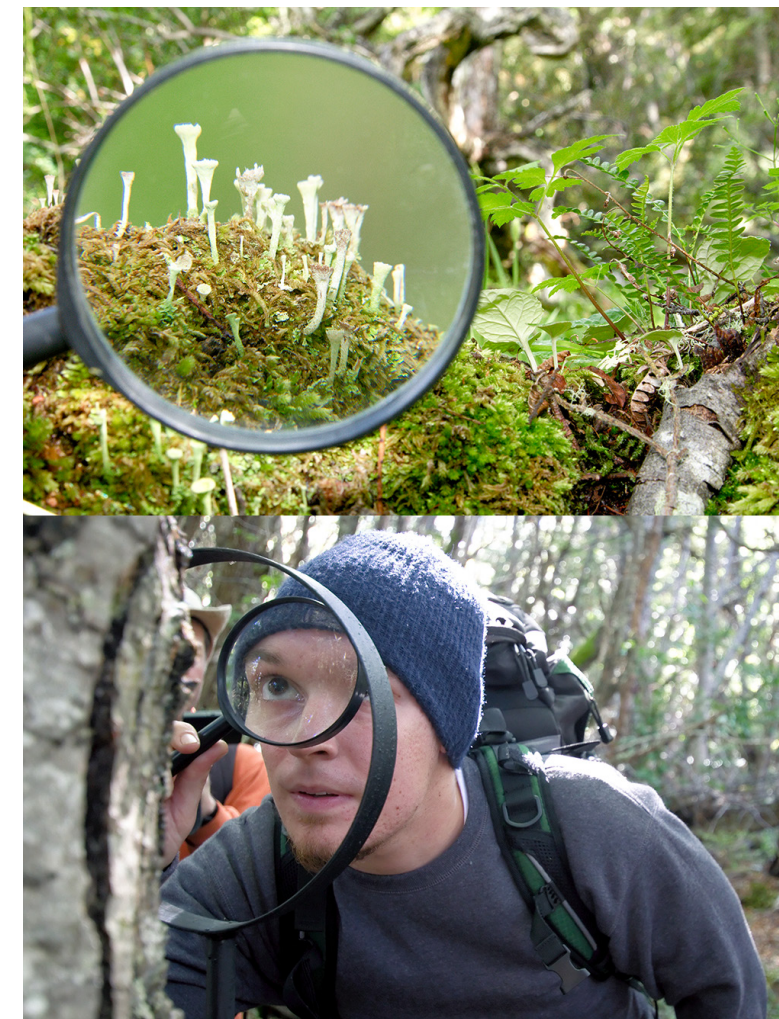

Figura 6. Práctica del ecoturismo con lupa para descubrir la belleza, diversidad e importancia ecológica de la pequeña flora de musgos, hepáticas y líquenes, y valorar las relaciones ecológicas, económicas, estéticas y éticas que la sociedad tiene con esta biodiversidad (Fotografías Adam Wilson).

líquenes y otros organismos asociados forman pequeños ecosistemas, y que los musgos y otros organismos son seres vivos. En cuanto tales, pueden ser considerados como co-habitantes con los seres humanos, y no solamente como "recursos naturales" a libre disposición para el uso sin regulación ni cuidado. El tercer paso de la FILAC incluye experiencias de campo de "encuentros directos" con los musgos y otros pequeños organismos (e.g., hongos, invertebrados) en su hábitat. A través de actividades de campo guiadas con una orientación ecológica y ética se estimuló, por ejemplo, la invención del "ecoturismo con lupa" (Fig. 6). Esta actividad contribuye a descubrir la belleza, diversidad e importancia socio-ecológica de esta pequeña flora que regularmente pasa desapercibida para los ciudadanos, profesores y tomadores de decisiones. El ecoturismo 
con lupa no sólo amplifica la visión sobre los musgos y otros organismos de los bosques en miniatura del Cabo de Hornos, sino que también provee una lupa metafórica que amplía nuestra imagen mental, perceptual y afectiva sobre la naturaleza y sobre nuestra relación con ella. La observación de la diversidad y la unidad de la vida, integra dimensiones éticas, estéticas y ecológicas que complementan la estrecha visión económica que prevalece actualmente en la relación de la sociedad contemporánea con la naturaleza. Finalmente, el cuarto paso de la FILAC implica la habilitación de espacios de conservación y educación. Por ejemplo, los estudiantes han participado en la creación del "Sendero de los Bosques en Miniatura" en el Parque Omora. Esta acción fomenta en los estudiantes un sentido de responsabilidad como ciudadanos educados ecológica- y éticamente. A través de su acción los estudiantes permiten a los visitantes observar y disfrutar de la diversidad de hábitats, las especies y las interacciones ecológicas, e invitan a diferentes instituciones y miembros de la sociedad a participar en la protección de la diversidad de hábitats y sus multifacéticas comunidades de pequeños $y$ grandes co-habitantes (Rozzi et al. 2012b). De esta manera, con la FILAC, en el Parque Omora se ha generado una institucionalidad que integra la investigación científica, la educación y el ecoturismo, al mismo tiempo que las ciencias, las artes y las humanidades.

\section{Trasfondo histórico y proyecciones del programa de investigación y educación del Parque Omora}

La experiencia en el Parque Omora demuestra la importancia vital de observar las riquezas ecológicas y culturales chilenas a macro y micro-escalas. La dimensión biofísica del enfoque biocultural es hoy ampliamente comprendida a través de la identificación de atributos únicos de la ecorregión subantártica de Magallanes (Rozzi et al. 2012a) y de las conexiones biogeográficas que explican las singularidades de su biodiversidad (Tabla 1). Menos comprendidas son las dimensiones culturales e institucionales del enfoque biocultural del Parque Omora. Estas dimensiones están intimamente vinculadas a acuñar nuevos nombres y a establecer nuevas instituciones, tales como un laboratorio natural que ha descubierto y acuñado un nombre para una ecorregión que era invisible (Tabla 3).

Para comprender el enfoque biocultural del programa de investigación y educación del Parque Omora y el futuro Centro Subantártico Cabo de Hornos es imprescindible realizar una revisión histórica de las distintas formas culturales de observar, comprender y valorar el territorio de la $\mathrm{RBCH}$ e institucionales de administrar este territorio. En la dimensión cultural, es interesante

Tabla 3. Aportes para la investigación y educación socio-ecológica a largo plazo en Cabo de Hornos hechos por el Programa de Conservación Biocultural Subantártico del Parque Omora desde su creación en el año 2000.

\begin{tabular}{|c|c|c|}
\hline Atributo biocultural & Año 2000 & Hoy \\
\hline Nombre de la Comuna & Navarino & Cabo de Hornos (2001) \\
\hline Presencia de la Universidad & $\begin{array}{l}\text { Baja } \\
\text { Presencia }\end{array}$ & Centro Universitario UMAG - Puerto Williams (2002) \\
\hline Nombre de la Región & Patagonia & Sub-Antártica de Magallanes (2002) \\
\hline $\begin{array}{l}\text { Reconocimiento de la } \\
\text { Biodiversidad }\end{array}$ & Pobre & $\begin{array}{l}\text { Hotspot mundial de diversidad de briófitas (musgos, hepáticas, líquenes) } \\
\text { (2003) }\end{array}$ \\
\hline $\begin{array}{l}\text { Reconocimiento por la } \\
\text { UNESCO }\end{array}$ & Inexistente & Reserva de Biosfera Cabo de Hornos, Chile (2005) \\
\hline $\begin{array}{l}\text { Relación científica con el } \\
\text { país }\end{array}$ & Inexistente & $\begin{array}{l}\text { Laboratorio Natural de Chile y co-fundador de Red LTSER-Chile (2008) } \\
\text { (Iniciativa Científica Milenio y CONICYT) }\end{array}$ \\
\hline $\begin{array}{l}\text { Relación científica con la } \\
\text { Antártica }\end{array}$ & Inexistente & $\begin{array}{l}\text { Parque Omora [ciencia subantártica] a } 950 \text { km de Base Escudero [ciencia } \\
\text { antártica] Isla Rey Jorge, Antártica Chilena (2013) }\end{array}$ \\
\hline Centro Científico Mundial & Inexistente & Futuro Centro Subantártico Cabo de Hornos (2014-2017) \\
\hline
\end{tabular}


notar cómo los relatos y las toponimias ancestrales y de los colonizadores europeos han ido introduciendo lenguajes y símbolos que expresan comprensiones y valoraciones diferentes del territorio denominado hoy como ecorregión subantártica de Magallanes. Para los pueblos originarios kawésqar y yagán, la toponimia se centra en los atributos bioculturales del lugar nombrado. Por ejemplo, los nombres de las bahías Puchachailgua, Cuaviquilgua y Alquilgua comparten el sufijo "gua" concepto geográfico kawésqar que denota un espacio resguardado para las embarcaciones en forma de ensenada, bahía o puerto. Los canales marinos que circundan la isla Navarino por el norte y el oeste eran llamados Onashaga y Yahgashaga, nombres yaganes que indican canales (shaga) aledaños a los territorios ona y yagán, respectivamente. En contraste, estos canales fueron renombrados por el capitán Fitzroy, respectivamente, como Canal Beagle en honor a su embarcación y Canal Murray en honor al contramaestre a bordo de su embarcación.

En su toponimia, los exploradores ingleses acuñaron nombres de lugares centrados en personajes destacados de sus expediciones o de la vida político-cultural de Inglaterra, tales como Cordillera Darwin. Los exploradores españoles, en cambio, proyectaron una toponimia que ilustra sobre todo la matriz católica-hispánica. En el siglo XVI, Hernando Gallego, Juan Ladrillero y Pedro Sarmiento de Gamboa, acuñaron nombres tales como de bahía San Alejo (1554), bahía de Nuestra Señora de los Remedios (1558), canal Trinidad (1579), isla Santa Inés (1580), cabo del Espíritu Santo (1580) y puerto de la Misericordia (1580) (Martinic, 2011). Las toponimias introducidas por exploradores españoles e ingleses coinciden, sin embargo, con el hecho que los nombres de los lugares suelen decir más acerca de la realidad cultural de los colonos que del territorio conquistado. El centro de atención y de la toponimia se desplaza hacia quienes otorgan los nombres, alejándose de los atributos particulares de los lugares que son nombrados. El nombre del Parque Omora procura reorientar esta tendencia al centrarse en un ave que tiene un papel ecológico y cultural clave, el picaflor chico (Sephanoides sephaniodes) u omora en lengua yagán (Rozzi et al. 2010c). El nombre que el programa de investigación del Parque Omora ha acuñado para el sudoeste de Sudamérica, ecorregión subantártica de Magallanes (sensu Rozzi, 2001), también es un término centrado en atributos biofísicos y culturales singulares.

La singularidad de la ecorregión subantártica de Magallanes quedaba eclipsada por la prevalencia de la imagen de la Patagonia. Por lo tanto, sus atributos como región lluviosa, montañosa y boscosa, habitada por pueblos canoeros marítimos y en los últimos cinco siglos por navegantes, exploradores europeos, pescadores y la Armada de Chile, eran menos visibles que aquellas que caracterizan a la estepa árida y plana, habitada por pueblos cazadores terrestres y en el período post-contacto europeo por gauchos y estancieros ganaderos y petroleros. El término ecorregión subantártica de Magallanes ha hecho visible a una región extrema o "de frontera" de Chile, y ha introducido un nuevo vocablo que hoy motiva programas de investigación en la Comisión Nacional de Ciencia y Tecnología (CONICYT), programas culturales y políticas de desarrollo territorial. El nombrar una ecorregión facilita el redescubrirla y luego conocerla, y aquello que se conoce se aprecia, se cuida y se conserva.

El programa de investigación y educación del Parque Omora procura descubrir atributos biofísicos y culturales de la zona de islas, canales, glaciares, fiordos, penínsulas, golfos y montañas de los archipiélagos al sur de Tierra del Fuego que contienen un diamante en bruto para la sociedad del siglo XXI. Para lograr el objetivo de pulir la comprensión acerca de esta joya natural, hoy menos ignota, pero más requerida de cuidado y valoración, es fundamental abordar la tercera dimensión del enfoque biocultural: la dimensión institucional. El carácter de "frontera" del Cabo de Hornos es un estímulo para pensar el presente y el futuro de esta región, desde el punto de vista institucional del Estado y de la universidad estatal regional, la Universidad de Magallanes (UMAG). Esta dimensión institucional ha conducido a la creación del Parque Omora, el Centro Universitario UMAG-Puerto Williams, y al futuro Centro Subantártico Cabo de Hornos, que generan una plataforma institucional para abordar desafíos y oportunidades que plantea el carácter de frontera de la $\mathrm{RBCH}$, que adquiere variadas dimensiones geográficas, naturales, culturales, económicas, administrativas y geopolíticas. 
Primero, la $\mathrm{RBCH}$ constituye una frontera de la civilización moderna y un refugio para la vida marina y terrestre por ser el maritorio y territorio más remoto permanentemente habitado en el Continente Americano, que ha sido incorporado tardíamente al "proceso civilizador" (Martinic, 2005, p. 15). En consecuencia, estas tierras archipelágicas adquieren hoy un carácter de refugio para una diversidad de modos de vida en este rincón austral del planeta, que es administrado por el Estado de Chile.

Segundo, la $\mathrm{RBCH}$ constituye una frontera geográfica y administrativa y un desafío para el Estado de Chile, puesto que su carácter geográficamente remoto plantea también un enorme desafío para la administración del territorio austral. A comienzos del siglo XX, la escasa presencia del Estado de Chile en las tierras al sur del canal Beagle, permitían la ocurrencia de delitos que violaban las leyes vigentes. En estas regiones extremas, geográficamente marginales, el Estado confrontaba así los límites de su capacidad de fiscalización; límites que impedían velar por el cumplimiento de la ley en la frontera administrativa del territorio de Chile. A mediados del siglo XX, esta situación comenzó a cambiar con el establecimiento de la Armada de Chile y la fundación de Puerto Luisa (futuro Puerto Williams en isla Navarino), los puestos de vigilancia naval en Puerto Banner (isla Picton), isla Lennox e islas Diego Ramírez, que representa un giro en la política administrativa. A partir de los años 1950s, se estableció un mayor control y cumplimiento de las leyes chilenas y hacia fines de los 1960s el Estado de Chile impulsó el Plan Navarino. Se revisaron y reordenaron las concesiones, se mejoró la infraestructura no sólo de Puerto Williams sino que también de Puerto Eugenia (isla Navarino), Caleta Piedra (isla Picton), Caleta Las Casas (isla Nueva), Caleta Cúter (isla Lennox), otras localices y se refundó Puerto Toro (isla Navarino). Al mismo tiempo, el Instituto de Fomento Pesquero prospectaba y evaluaba la riqueza de la biota marina, y en tierra se llevaban a cabo prospecciones mineras. El historiador Mateo Martinic (2005) ha concluido que en la década de los 1970s "las islas australes formaban ya indisoluble y definitivamente parte integrante del cuerpo vivo y activo de la República” (p. 186).

Tercero, la $\mathrm{RBCH}$ constituye una frontera del conocimiento científico y un desafío para superar la condición de Terra Incognita Australis. Este desafío es abordado por la UMAG. Para la comprensión del momento histórico actual, y del valor (potencial y actual) que presenta el Cabo de Hornos en el mundo globalizado de hoy, cobra especial relevancia la actividad científica en el territorio, en cuanto permite re-descubrir un "tesoro natural". La historia de "descubrimiento y reconocimiento científico del territorio" incluye destacadas expediciones, como aquellas del capitán James Cook (1769 y 1774), el biólogo Charles Darwin (1832-1833), la Misión Científica Francesa (1882-1883) y las de los etnólogos Martín Gusinde y Wilhem Koppers (1918, 1920, 1923). El valor natural y cultural albergado por el territorio al sur del canal Beagle fue oportunamente reconocido por el gobierno chileno en el año 1945 con "una previsora disposición administrativa del gobierno del Presidente Juan Antonio Ríos, quien hizo posible la creación del Parque Nacional Cabo de Hornos, de 63.000 hectáreas de superficie, el primero en su clase en la jurisdicción territorial de Magallanes (D.S. 995 del Ministerio de Tierras y Colonización de fecha 25-VII-1945)...[para proteger] la condición de absoluta pristinidad del archipiélago de ese nombre, de la especificidad de sus ecosistemas naturales y su situación marginal en el extremo meridional de América" (Martinic, 2005, p. 258). Esta cita del historiador introduce lúcidamente el concepto de un "tesoro natural redescubierto", quien concluye que el fruto más trascendente de este redescubrimiento del tesoro natural corresponde a la "iniciativa de concebir y proponer la creación de la Reserva de Biosfera Cabo de Hornos a la UNESCO" (Martinic, 2005, p. 261).

Hoy el Estado de Chile ha reafirmado su compromiso con la $\mathrm{RBCH}$ a través de un ordenamiento territorial que procura el bien común, a través del desarrollo sustentable y la conservación del patrimonio natural y cultural de Cabo de Hornos para todos los habitantes de la región y el planeta. Martinic (2005) destaca que "la especificidad excepcional de la biodiversidad que habita en el archipiélago Cabo de Hornos, valorada por la ciencia universal y reconocida por el prestigioso organismo internacional (UNESCO), permite un uso turístico especializado... un tesoro 
de pristinidad virtualmente único, de los que en diferente variedad quedan poquísimos en la Tierra. Esta motivación científica, suficiente de suyo, aún se enriquece con la carga de historia acumulada a lo largo de los siglos y con la herencia cultural indígena recuperada" (p. 261). La labor científica de la UMAG en la $\mathrm{RBCH}$, iniciada con estudios precursores en el archipiélago Cabo de Hornos a fines de los 1970s y comienzos de los 1980s, se ha consolidado con la creación del Centro Universitario UMAG-Puerto Williams y el Parque Etnobotánico Omora, concebido como un laboratorio natural y aula natural.

Cuarto, la $\mathrm{RBCH}$ constituye una frontera de la economía y un desafío para fomentar la sustentabilidad ambiental y social. Bajo una perspectiva ecológica y económica planteada en el proceso de creación de la $\mathrm{RBCH}$, el valor de conservar el patrimonio cultural y natural plantea que "En términos de rentabilidad podría afirmarse que Chile y la humanidad han hecho la mejor inversión al conservar estos archipiélagos de tundra, fiordos y glaciares casi inalterados. Ninguna tasa de interés igualaría el aumento de valor que ha cobrado el capital natural del territorio del Cabo de Hornos en su estado virgen, remoto, pacífico y singular a comienzos del siglo XXI; cuando prevalece una sociedad globalmente homogeneizada, violenta $e$ insegura, y con una aspiración creciente por lugares diferentes o alternativos" (Rozzi, 2004, p. 12). Esta cita del primer documento enviado a UNESCO para postular a la $\mathrm{RBCH}$, propone preservar, con fines de conservación y sustentabilidad económica, el Parque Nacional Cabo de Hornos como "zona núcleo" definida en la zonificación de la $\mathrm{RBCH}$ diseñada por el Gobierno de Chile y aprobada por UNESCO. El Parque Nacional Cabo de Hornos es hoy un lugar único en el mundo, una frontera al desarrollo económico donde todavía es posible experimentar la aventura, el riesgo de navegar hacia la cima austral del Nuevo Mundo, donde todavía es necesario esperar días de vientos calmos para zarpar y donde la naturaleza aún puede ser vivenciada al margen del "control tecnológico". La zonificación de la $\mathrm{RBCH}$ indica que la infraestructura debe ubicarse en la isla Navarino y otros sectores de la zona de transición. La oportunidad para conservar áreas núcleos donde vivenciar una naturaleza más allá de la frontera de homogeneización biocultural representa una oportunidad para una sabia inversión económica que nos ofrece esta pequeña cúspide austral, hito geográfico que le ha tocado administrar a Chile.

Hoy, el país y el mundo reconocen y observan la $\mathrm{RBCH}$ y valoran su diversidad biológica y cultural. La integración de la ecología y la ética en metodologías de conservación biocultural, junto a las singularidades biológicas de la ecorregión subantártica de Magallanes, concitan el interés mundial. Chile es reconocido por los observatorios astronómicos y su "telescopios" para explorar el cosmos desde Atacama, al mismo tiempo que por las "lupas" para explorar la exuberante diversidad florística y los valores éticos entre la sociedad humana y la biodiversidad desde el Cabo de Hornos. Esta analogía establecida metafóricamente por investigadores del Parque Omora (Rozzi, 2014), se materializará en la creación del futuro Centro Subantártico Cabo de Hornos. El centro estará organizado en tres módulos que aportarán en tres áreas de investigación (teórica y aplicada) y educación interdisciplinaria.

(i) Un centro de visitantes para recibir $y$ orientar a los turistas que arriben a la $\mathrm{RBCH}$. El Centro Subantártico Cabo de Hornos cumplirá un papel similar al desempeñado por la Estación Charles Darwin en la Reserva de la Biosfera Galápagos en Ecuador, generando nuevas temáticas turísticas tales como el ecoturismo con lupa o la Ruta de Darwin en Cabo de Hornos (Tabla 2), y ofreciendo un lugar donde grupos de turistas que arriben en cruceros y otros medios puedan tener una introducción para apreciar los atributos de la $\mathrm{RBCH}$.

(ii) Un centro de excelencia en investigación científica y filosófica que innovará a nivel mundial en la integración de estas disciplinas en una ética biocultural para reorientar a la sociedad nacional y global hacia la sustentabilidad de la vida. El Centro Subantártico Cabo de Hornos cumplirá esta función a través de una estrecha colaboración con una red de instituciones regionales, nacionales e internacionales, en particular con la Red LTSERChile y la Red ILTER.

(iii) Un Centro de Formación Técnica en 
Turismo de Intereses Especiales que incluirá tres áreas de especialización: gastronomía regional, áreas protegidas y turismo científico. El enfoque biocultural innovará a nivel mundial en la integración de las ciencias y la ética en el turismo sustentable. La región biogeográfica también será un aporte clave al incluir en la oferta internacional una región que había permanecido invisible debido a la falta de un nombre que la distinguiera de la Patagonia, y al énfasis que el turismo de naturaleza ha puesto en regiones tropicales y últimamente en la Antártica. El turismo científico subantártico de Magallanes es diferente también del turismo subantártico de Nueva Zelanda, Australia y Sudáfrica, puesto que las islas subantárticas de esos países carecen de bosques.

En suma, para conocer y cuidar la $\mathrm{RBCH}$, más allá de la frontera de homogenización biocultural global el programa de investigación y educación del Parque Omora nos estimula a repensar y evaluar los proyectos de desarrollo para la región, el país y el mundo. Para la sociedad global del siglo XXI, sumida en una espiral de agitación bélica, desigualdades e injusticias sociales, modos de vivir cada vez más uniformes e insensibles a la diversidad de formas de vida y culturas que cohabitan en el planeta, la $\mathrm{RBCH}$ representa un hito natural y cultural que se conserva en condiciones de paz y con expresiones de vida que no han sido acalladas por la uniformidad de la ola de homogeneización biocultural. Desde esta frontera en la cima austral de América, el futuro Centro Subantártico Cabo de Hornos permitirá elaborar análisis críticos de los modelos de desarrollo desplegados durante los dos últimos siglos, para debatir y concebir nuevos modos de vida sustentable que procuren el bienestar de los diversos seres humanos y otros seres que habitamos al sur del mundo. Para lograr este objetivo, a nivel nacional $e$ internacional se trabajará con la red de reservas de la biosfera de UNESCO. A nivel regional, esta tarea se abordará en estrecha colaboración con los centros universitarios UMAG de Puerto Natales, Punta Arenas y Porvenir. Mientras en el Parque Nacional Torres del Paine se desarrollan grandes esfuerzos de restauración ecológica, en la $\mathrm{RBCH}$ se diseña el entro Subantártico Cabo de Hornos para consolidar un programa de investigación y educación biocultural que contribuya a la conservación de un tesoro natural y al bienestar de los co-habitantes, humanos y no-humanos, de la cumbre austral del Continente Americano.

\section{AGRADECIMIENTOS}

Agradecemos a las numerosas personas $e$ instituciones que han apoyado en la creación de la Reserva de la Biosfera Cabo de Hornos y que han colaborado con el Parque Etnobotánico Omora en su labor de investigación y educación durante la última década. Este manuscrito se basa en los resultados y trabajo del equipo de investigación del Parque Omora. Francisca Massardo y Jaime J. Jiménez han hecho valiosos comentarios al texto. Este trabajo fue apoyado por los proyectos CONICYT+PAI (Concurso nacional Inserción en la academia, convocatoria 2014 + Folio 791400024, patrocinado por Andrés Mansilla) e ICM P05-002 y Basal-CONICYT PFB-23. Este trabajo es una contribución del Programa de Conservación Biocultural Subantártica, coordinado por la Universidad de Magallanes y el Instituto de Ecología y Biodiversidad de Chile en colaboración con University of North Texas, EE.UU.

\section{LITERATURA CITADA}

Aguirre, J. F. (2015). Hermeneutics and field environmental philosophy: integrating ecological sciencies and ethics into earth stewardship. In R. Rozzi, F. S. Chapin, J. B. Callicott, S. T. A. Pickett, M. E. Power, J. J. Armesto and R. H. May Jr. (Eds), Earth Stewardship: Linking ecology and ethics in theory and practice (pp. 235247). Dordrecht: Springer.

Arango, X., Rozzi, R., Massardo, F., Anderson, C. B., \& Ibarra, T. (2007). Discovery and implementation of Magellanic woodpecker (Campephilus magellanicus) as an emblematic species: A biocultural approach for conservation in Cape Horn Biosphere Reserve. Magallania, 35(2), 71-88.

Armesto, J., Arroyo, M. K., \& Villagrán, C. (1980). Altitudinal distribution, cover and size structure of umbelliferous cushion 
plants in the high Andes of central Chile. Oecologia Generalis, 1(14), 327-332.

Armesto, J. J., Rozzi, R., Smith-Ramírez, C., \& Arroyo, M. T. K. (1998). Effective conservation targets in South American temperate forests. Science, 282, 12711272.

Armesto, J. J., Núñez, M, Donoso P., \& Rozzi, R. (2014). Introducción a la Sección Especial de Revista Bosque. Avances de una red de sitios de estudios ecológicos a largo plazo en el suroeste de Sudamérica. Bosque, 35, 413-414.

Arróniz-Crespo, M., Pérez-Ortega, S., de los Ríos, A., Green, T. G. A., Ochoa-Hueso, R., Casermeiro, M. A., de la Cruz, M. T.,...Sancho, L. G. (2014). Bryophytecyanobacteria associations during primary succession in recently deglaciated areas of Tierra del Fuego (Chile). PLoS ONE 9(9): e108759. doi: 10.1371/journal. pone.0108759

Arroyo, M. K., Cavieres, L., Peñaloza, A., Riveros, M., \& Faggi, A. M. (1996). Relaciones fitogeográficas y patrones regionales de riqueza de especies en la flora del bosque lluvioso templado de Sudamérica. In: J.J. Armesto, C. Villagrán \& M.K. Arroyo (Eds), Ecología de los Bosques Nativos de Chile (pp. 71-92). Santiago, Chile: Editorial Universitaria.

Arroyo, M. T. K., Rozzi, R., Simonetti, J. A., Marquet, P., \& Salaberry, M. (2000). Central Chile. In A. Mittermeier, P. RoblesGil \& C. Goettsch-Mittermeier (Eds.). Hotspots: Earth's biologically wealthiest and most threatened ecosystems (pp. 210-231). Ciudad de México: Cemex.

Berghöfer, U., Rozzi, R., \& Jax, K. (2008). Diverse Perspectives on Nature in the Cape Horn Biosphere Reserve. Environmental Ethics, 30, 273-294.

Berghöfer, U., Rozzi, R., \& Jax, K. (2010). Many eyes on nature: diverse perspectives in the Cape Horn Biosphere Reserve and their relevance for conservation. Ecology and Society,15, 18: http://www. ecologyandsociety.org/vol15/iss1/art18/ Carmona, M. R., Aravena, J. C., Bustamante-
Sánchez, M. A., Celis-Diez, J. L., Charrier, A., Díaz, I. A.,...Armesto, J. J. (2010). Estación Biológica Senda Darwin: Investigación Ecológica De Largo plazo en la interfase ciencia-sociedad. Revista Chilena de Historia Natural, 83, 113142.

Chapin, M. (2004). A challenge to conservationists. World Watch, 17, 1731.

Contador, T.A. (2011). Benthic macroinvertebrates of temperate, sub-Antarctic streams. Tesis doctoral. Denton, EE.UU: Department of Biological Sciences, University of North Texas.

Contador, T., Kennedy, J. H., \& Rozzi, R. (2012). The conservation status of southern South American aquatic insects in the literature. Biodiversity and Conservation, 21, 2095-2107.

Contador, T., Kennedy, J, Ojeda, J., Feinsinger, P., \& Rozzi, R. (2014). Ciclos de vida de insectos dulceacuícolas y cambio climático global en la ecorregión subantártica de Magallanes: investigaciones ecológicas a largo plazo en el Parque Etnobotánico Omora, Reserva de Biosfera Cabo de Hornos (55º). Bosque, 35, 429-437.

Couve, E., \& Vidal, C. (2003). Birds of Patagonia, Tierra del Fuego \& Antarctic Peninsula. Punta Arenas: Editorial Fantástico Sur Birding Ltda.

Crego, R. D., Jiménez, J. E., Soto, C., Barroso, O., \& Rozzi, R. (2014). Tendencias poblacionales del visón norteamericano invasor (Neovison vison) y sus principales presas nativas desde su arribo a isla Navarino, Chile. Boletín de la Red Latinoamericana para el Estudio de Especies Invasoras, 4, 4-18.

Crego, R. D., Jiménez, J. E. \& Rozzi, R. (2015). Expansión de la invasión del visón norteamericano (Neovison vison) en la Reserva de la Biosfera Cabo de Hornos, Chile. Anales del Instituto de la Patagonia, 43, 157-162.

Dollenz, O. (1991). Recolonización de un coironal incendiado en el Parque Nacional Torres del Paine, Magallanes, 
Chile. Master Thesis, Facultad de Ciencias, Santiago, Chile: Universidad de Chile.

Donoghue, M. J. (2011). Bipolar biogeography. Proceedings of the National Academy of Sciences, 108(16), 6341-6342.

Goffinet, B., Rozzi, R., Lewis, L., Buck, W., \& Massardo, F. (2012). Los Bosques en Miniatura del Cabo de Hornos: Ecoturismo con Lupa. Denton y Punta Arenas: UNT Press-Ediciones Universidad de Magallanes.

Guevara, S., \& Laborde, J. (2008). The Landscape Approach: Designing New Reserves for Protection of Biological and Cultural Diversity in Latin America. Environmental Ethics, 30(3), 251-262.

Gutiérrez, J. R., Meserve, P. L., Kelt, D. A., Engilis Jr, A., \& Andrea, M. (2010). Long-term research in Bosque Fray Jorge National Park: Twenty years studying the role of biotic and abiotic factors in a Chilean semiarid scrubland. Revista Chilena de Historia Natural, 83(1), 6998.

Haberl, H., Graube, V., Díaz-Delgado, R., Krauze, K., Neuner, A., Peterseil, J., Plutzar, C. Singh, S. J., \& Vadineanu, A. (2009). Towards an integrated model of socioeconomic biodiversity drivers, pressures and impacts: a feasibility study based on three European long-term socioecological research platforms. Ecological Economics, 68, 1797-1812.

Heller, N. E., \& E. S. Zavaleta. (2009). Biodiversity management in the face of climate change: A review of 22 years of recommendations. Biological Conservation, 142,14-32.

Ibarra, J. T., Fasola, L., Macdonald, D. W., Rozzi, R., \& Bonacic, C. (2009). Invasive American mink in wetlands of the Cape Horn Biosphere Reserve, Southern Chile: what are they eating? Oryx, 43, 87-90.

Ibarra, J. T., Schüttler, E., McGehee, S., \& Rozzi, R. (2010). Tamaño de puesta, sitios de nidificación y éxito reproductivo del caiquén (Chloephaga picta Gmelin, 1789) en la Reserva de Biosfera Cabo de Hornos, Chile. Anales del Instituto de la Patagonia, 38, 73-82.
Ibarra, J. T., Barreau, A., Massardo, F., \& Rozzi, R. (2012). El cóndor andino: una especie biocultural clave del paisaje sudamericano. Boletín Chileno de Ornitología, 18, 1-22.

Jax, K. (2001). Naturbild, Ökologietheorie und Naturschutz: zur Geschichte des Ökosystemmanagements im YellowstoneNationalpark. Verhandlungen der Gesellschaft für Geschichte und Theorie der Biologie, 7, 115-134.

Jax, K., \& Rozzi, R. (2004). Ecological theory and values in the determination of conservation goals: examples from temperate regions of Germany, United States of America, and Chile. Revista Chilena de Historia Natural, 77, 349-366.

Jiménez, J. E., Crego, R. D., Soto, G. E., Román, I., Rozzi, R., \& Vergara, P. M. (2014). Potential impact of the alien American mink (Neovison vison) on Magellanic woodpeckers (Campephilus magallanicus) in Navarino Island, southern Chile. Biological Invasions, 16, 961-966.

Jofre, J., Massardo, F., Rozzi, R., Goffinet, B., Marino, P., Raguso, R., \& Navarro, N. P. (2010). Fenología de Tayloria dubyi (Splachnaceae) en las turberas de la Reserva de Biosfera Cabo de Hornos. Revista Chilena de Historia Natural, 83, 195-206.

Jofre, J., Goffinet, B., Marino, P., Raguso, R. A., Nihei, S. S., Massardo, F., \& Rozzi, R. (2011). First evidence of insect attraction by a Southern Hemisphere Splachnaceae: The case of Tayloria dubyi in the Reserve Biosphere Cape Horn, Chile. Nova Hedwigia, 92, 317-326.

Lewis, L. R., Rozzi, R., \& Goffinet, B. (2014a). Direct long-distance dispersal shapes a New World amphitropical disjunction in the dispersal-limited dung moss Tetraplodon (Bryopsida: Splachnaceae). Journal of Biogeography, 41, 2385-2395.

Lewis, L. R., Behling, E., Gousse, H., Qian, E., Elphick, C.S., Lamarre, J.F., Bêty, J.,...Goffinet, B. (2014b). First evidence of bryophyte diaspores in the plumage of transequatorial migrant birds. PeerJ 
2:e424: https://dx.doi.org/10.7717/ peerj. 424

Li, B., Parr, T., \& Rozzi, R. (2015). Geographical and Thematic Distribution of Publications Generated at the International Long-Term Ecological Research Network (ILTER) Sites. In: R. Rozzi, F. S. Chapin, J.B. Callicott, S.T.A. Pickett, M.E. Power, J.J. Armesto and R.H May Jr. (Eds), Earth Stewardship: Linking ecology and ethics in theory and practice (pp. 195-216). Dordrecht: Springer.

Lui, J., Dietz, T., Carpenter, S.R., Alberti, M., Folke, C., Moran E., Pell, A.C.,...Taylor, W. W. (2007). Complexity of coupled human and natural systems. Science, 317, 1513-1516.

Mansilla A., Ávila M., \& Yokoya, N. S. (2012). Current knowledge on biotechnological interesting seaweeds from the Magellan Region, Chile. Revista Brasileira de Farmacognosia, 22, 760-767.

Mansilla, A., Ávila, A., Ramírez, M. E., Ojeda, J., Rodriguez, J. P,. Rosenfeld, S., \& Marambio, J. (2013a). Macroalgas marinas bentónicas del submareal somero de la ecorregión subantártica de Magallanes, Chile. Anales del Instituto de la Patagonia, 41, 49-62.

Mansilla, A., González, L., \& Astorga, M. S. (2013b). Uso de algas marinas en la gastronomía magallánica. Punta Arenas: Ediciones Universidad de Magallanes.

Mansilla, A., Rodriguez, J. P., Souza, J. M., Rosenfeld, S., Ojeda, J., \& Yokoya, N. S. (2014a). Growth responses to temperature, salinity and nutrient variations, and biomass variation and phenology of Ahnfeltia plicata (Rhodophyta, Ahnfeltiales): a commercially interesting agarophyte from the Magellanic Region, Chile. Journal of Applied Phycology, 26(2), 1133-1139.

Mansilla, A., Rosenfeld, S., Rendoll, J., Murcia, S., Werlinger, C., Yokoya, N. S., \& Terrados, J. (2014b). Tolerance response of Lessonia flavicans from the subAntarctic ecoregion of Magallanes under controlled environmental conditions. Journal of Applied Phycology, 26(5),
1971-1977.

Martinic, M. (1984). Última esperanza en el tiempo. Punta Arenas, Chile: Ediciones Universidad de Magallanes.

Martinic, M. (2005). Crónica de las tierras al sur del canal Beagle. Punta Arenas, Chile: Ediciones Hotel Lakutaia.

Martinic, M. (2011). El occidente fueguino todavía una incógnita. Punta Arenas, Chile: La Prensa Austral.

Massardo, F., Dollenz, O., \& Rozzi, R. (2001). Ecoturismo en el Cono Austral de América. In R. Primack, R. Rozzi, P. Feinsinger, R. Dirzo\& F. Massardo (Eds.), Elementos de conservación biológica: perspectivas Latinoamericanas (pp. 303-305). Ciudad de México: Fondo de Cultura Económica.

Massardo, F., \& Rozzi, R. (2006). The world's southernmost ethnoecology: Yahgan craftsmanship and traditional ecological knowledge. Punta Arenas, Chile: Ediciones Universidad de Magallanes.

Massardo, F., Mansilla, A., Armesto, J. J., \& Rozzi, R. (2012). Ecología y Filosofía Ambiental desde el Confín Austral de América. Environmental Ethics, 34, 7-8.

Medina, Y. (2013). Bosques en Miniatura del Cabo de Hornos: el Turismo con Lupa como Herramienta para la Educación, Conservación y Turismo Científico en la Ecorregión Subantártica de Magallanes. Tesis de Magíster en Ciencias. Punta Arenas, Chile: Universidad de Magallanes.

Méndez, M. (2013). Asociaciones Positivas de Plantas Vasculares y Musgos a Bolax gummifera (Lam.) Spreng. en Comunidades Alto-andinas de la Isla Navarino. Tesis de Magíster en Ciencias. Concepción, Chile: Universidad de Concepción.

Méndez, M., Rozzi, R., \& Cavieres, L. (2013). Flora vascular y no-vascular en la zona altoandina de la isla Navarino $\left(55^{\circ} \mathrm{S}\right)$, Reserva de biosfera Cabo de Hornos, Chile. Gayana Botánica, 70, 337-343.

Merino, S., Moreno, J., Vásquez, R., Martínez, J., Sánchez-Monsálvez, I., Estades, C. F., Ippi, S.,...McGehee, S. (2008). Haematozoa in forest birds from southern 
Chile: Looking for latitudinal gradients in prevalence and parasite lineage richness. Austral Ecology, 33, 329-340.

Mittermeier, R. A., Mittermeier, C. G., Brooks, T. M., Pilgrim, J. D., Konstant, W. R., da Fonseca, G. A. B., \& Kormos, C. (2003). Wilderness and biodiversity conservation. Proceedings of the National Academy of Sciences, 100, 10309-10313.

Navarro, R. M., Olave, F., Hayas, A., \& Castillo, M. (2015). Metodología para la elaboración de un plan de restauración postincendio en Chile: la experiencia del Parque Nacional de Torres del Paine. Anales del Instituto de la Patagonia, 43, 53-73.

Ohl, C., Krauze, K., \& Grünbühl, C. (2007). Towards an understanding of longterm ecosystem dynamics by merging socioeconomic and environmental research: Criteria for long-term socio-ecological research sites selection. Ecological Economics, 63, 383-391.

Ojeda J. (2013). Dinámica estacional de macroalgas y moluscos intermareales en canales subantárticos del Cabo de Hornos: Una aproximación biocultural desde la filosofía ambiental de campo. Tesis de Magíster en Ciencias. Punta Arenas, Chile: Universidad de Magallanes.

Ojeda, J., Marambio, J., Rosenfeld, S., Rozzi, R., \& Mansilla, A. (2014). Patrones estacionales y espaciales de la diversidad de moluscos intermareales de bahía Róbalo, canal Beagle, Reserva de la Biosfera Cabo de Hornos, Chile. Revista de Biología Marina y Oceanografía, 49, 493-509.

Ojeda, J., Marambio, J., Rosenfeld, S., Contador, T., Rozzi, R., \& Mansilla, A. (2015). Seasonal macroalgal assemblages in Magallanic sub-Antarctic channels: A case study in the rocky intertidal of Róbalo Bay (5455'S), Beagle Channel, Chile. Polar Biology: en prensa.

Palmer, C. (2013). Contested Frameworks in Environmental Ethics. In Linking Ecology and Ethics for a Changing World. In R. Rozzi, S. T. A. Pickett, C. Palmer, J. J. Armesto \& J. B. Callicott (Eds.), Linking Ecology and Ethics for a Changing
World: Values, Philosophy, and Action, Ecology and Ethics (pp. 191-206). Dodrecht: Springer.

Parr, T. W., Ferretti, M., Simpson I. C., Forsius, M., \& Kóvacs-Láng, E. (2002). Towards a long-term integrated monitoring programme in Europe: Network design in theory and practice. Environmental Monitoring and Assessment, 78, 253290.

Pizarro, M., \& Caballero, P. (2011). Diversicuentos. Cuentos infantiles sobre la diversidad biocultural subantártica. Punta Arenas.

Poole, A. K., Hargrove, E. C., Day, P., Forbes, W., Berkowitz, A. R., Feinsinger, P., \& Rozzi, R. (2013). A Call for Ethics Literacy in Environmental Education. In R. Rozzi,S. T. A. Pickett, C. Palmer, J. J. Armesto \& J. B. Callicott (Eds.). Linking Ecology and Ethics for a Changing World: Values, Philosophy, and Action, Ecology and Ethics (pp. 349-372). Dodrecht: Springer.

Popp, M., Mirré, V., \& Brochmann, C. (2011). A single mid-Pleistocene long-distance dispersal by a bird can explain the extreme bipolar disjunction in crowberries (Empetrum). Proceedings of the National Academy of Sciences USA, 108, 65206525.

Redman, C. L., Grove, J. M., \& Kuby, L. H. (2004). Integrating social science into the long-term ecological research (LTER) network: Social dimensions of ecological change and ecological dimensions of social change. Ecosystems, 7, 161-171.

Repetto, F., \& Cabello, J. (2015). Potencial de restauración ecológica en zonas de uso público en el Parque Nacional Torres del Paine. Anales del Instituto de la Patagonia 43, 115-121.

Reyes, R., Jiménez, J. E., \& Rozzi, R. (2015). Daily patterns of activity of a Magellanic Subantarctic forests bird ensemble at Omora Park (55ㅇ), Cape Horn Biosphere Reserve, Chile. Polar Biology, 38, 401411.

Rozzi, R. (2001). Biological and cultural conservation in the archipelago forest 
ecosystems of southern Chile. Ph.D. Dissertation. Storrs, Connecticut, USA: Department of Ecology and Evolutionary Biology, University of Connecticut.

Rozzi, R. (2004). El territorio insular de Cabo de Hornos: Un tesoro biocultural al sur del mundo. In: Rozzi, R. Massardo, F. \& Anderson, C. (Eds), Reserva de Biosfera Cabo de Hornos: Una propuesta de conservación y turismo para el desarrollo sustentable en el extremo austral de América (pp. 11-25). Ediciones de la Universidad de Magallanes: Punta Arenas, Chile.

Rozzi, R. (2012a). Biocultural ethics: the vital links between the inhabitants, their habits and regional habitats. Environmental Ethics, 34, 27-50.

Rozzi, R. (2012b). South American Environmental Philosophy: Ancestral Amerindian Roots and Emergent Academic Branches. Environmental Ethics, 34, 343-365.

Rozzi, R. (2013). Biocultural Ethics: From Biocultural Homogenization toward Biocultural Conservation. In R. Rozzi, S. T. A. Pickett, C. Palmer, J. J. Armesto \& J. B. Callicott (Eds.). Linking Ecology and Ethics for a Changing World: Values, Philosophy, and Action, Ecology and Ethics (pp. 9-32). Dodrecht: Springer.

Rozzi, R. (2014). Hacia una Ética Biocultural Planetaria Interregional: Aproximaciones, Metodologías y Propuestas desde el Sur del Mundo. Actas II Encuentro Observando a Chile desde la Distancia, pp. 31-41. Barcelona, España: Ediciones Universidad de Barcelona.

Rozzi, R., Silander, J., Armesto, J. J., Feinsinger, P., \& Massardo, F. (2000). Three levels of integrating ecology with the conservation of South American temperate forests: the initiative of the Institute of Ecological Research Chiloé, Chile. Biodiversity and Conservation, 9(8), 1199-1217.

Rozzi, R., \& Heidinger, K. (2006). The route of Darwin through the Cape Horn Archipelago. Punta Arenas, Chile: Ediciones Universidad de Magallanes.

Rozzi, R., Massardo, F., Berghöfer, A., Anderson,
C., Mansilla, A., Mansilla, M., Plana, J.,... Araya, P. (2006a). La Reserva de Biosfera Cabo de Hornos. Punta Arenas, Chile: Ediciones Universidad de Magallanes.

Rozzi, R., Massardo, F., Anderson, C.,Heidinger, K., \& Silander, J. Jr. (2006b). Ten principles for biocultural conservation at the southern tip of the Americas: The approach of the Omora Ethnobotanical Park. Ecology and Society, 11, 43: http://www. ecologyandsociety.org/vol11/iss1/art43/

Rozzi, R., Massardo, F., Mansilla, A., Anderson, C. B., Berghöfer, A., Mansilla, M., Gallardo M.R.,...Barros, E. (2007). La reserva de biosfera Cabo de Hornos: un desafío para la conservación de la biodiversidad e implementación del desarrollo sustentable en el extremo austral de América. Anales del Instituto de la Patagonia, 35, 55-70.

Rozzi, R., Arango, X., Massardo, F., Anderson, C., Heidinger, K., \& Moses, K. (2008a). Field Environmental Philosophy and Biocultural Conservation: The Omora Ethnobotanical Park Educational Program. Environmental Ethics, 30, 325-336.

Rozzi, R., Armesto, J., Goffinet, B., Buck, W., Massardo, F., Silander, J., Kalin-Arroyo, M.,...Callicott, J. B. (2008b). Changing lenses to assess biodiversity: patterns of species richness in sub-Antarctic plants and implications for global conservation. Frontiers in Ecology and the Environment, 6, 131-137.

Rozzi, R., Massardo, F., Cruz, F., Grenier, C., Muñoz, A., Mueller, E., \& Elbers, J. (2010a). Galapagos and Cape Horn: ecotourism or greenwashing in two emblematic Latin American archipelagoes? Environmental Philosophy, 7, 1-32.

Rozzi, R., Anderson, C. B., Pizarro, J. C., Massardo, F., Medina, Y., Mansilla, A., Kennedy, J.H.,...Kalin, M.T. (2010b). Filosofía ambiental de campo y conservación biocultural en el Parque Etnobotánico Omora: aproximaciones metodológicas para ampliar los modos de integrar el componente social ("s") en Sitios de Estudios Socio-Ecológicos a Largo Plazo (SESELP). Revista Chilena 
de Historia Natural, 83, 27-68.

Rozzi, R., Massardo, F., Anderson, C., McGehee, S., Clark, G., Egli, G., Ramilo, E.,... Zárraga, C. (2010c). Multi-Ethnic Bird Guide of the Sub-Antarctic Forests of South America. UNT Press - Ediciones Universidad de Magallanes, Denton TX and Punta Arenas, Chile.

Rozzi, R., Massardo, F., Medina, Y., Moses, K., Caballero, P., Camelio, E., Morales, V., Lewis, L.,...Russell, S. (2010d). Ecoturismo con lupa: integración de las ciencias ecológicas y la ética ambiental. Ambiente y Desarrollo, XXIV, 30-37.

Rozzi, R., Armesto, J. J., Gutiérrez, J., Massardo, F., Likens, G., Anderson, C. B., Poole, A...Kalin, M. T. (2012a). Integrating ecology and environmental ethics: Earth stewardship in the southern end of the Americas. BioScience, 62, 226-236.

Rozzi, R., Lewis, L., Massardo, F., Medina, Y., Moses, K., Méndez, M., Sancho, L.,... Goffinet, B. (2012b). Ecoturismo con Lupa en el Parque Omora. Punta Arenas: Ediciones Universidad de Magallanes.

Rozzi, R., \& Schüttler, E. (2013). Protecting a land of fire and ice. A World of SCIENCE, 11, 23-27.

Rozzi, R., \& Jiménez, J. E. (2014). Ornitología Subantártica de Magallanes: Primera Década de Estudios de Aves en el Parque Etnobotánico Omora, Reserva de Biosfera Cabo de Hornos. Denton y Punta Arenas: UNT Press-Ediciones Universidad de Magallanes.

Rozzi, R., Massardo, F., Contador, T., Crego, R. D., Méndez, M., Rijal, R., Cavieres, L., \& Jiménez, J. E. (2014). Filosofía ambiental de campo: ecología y ética en las redes LTER-Chile e ILTER. Bosque, 35, 439447.

Rozzi, R., Chapin, S. F., Callicott, J. B., Pickett, S. T. A., Power, M., Armesto, J. J., \& May Jr., R. H. (2015). Earth Stewardship: Linking Ecology and Ethics in Theory and Praxis. Dodrecht: Springer.

Runte, A. (1997). National parks. The American experience. Nebraska: University of Nebraska Press.
Sherriffs, M., Ippi, S., Anderson, C., Rozzi, R., \& Zúñiga, A. (2005). Explorando la Microbiodiversidad del Cabo de Hornos. Punta Arenas, Chile: Ediciones Universidad de Magallanes.

Schüttler, E., Cárcamo, J., \& Rozzi, R. (2008). Diet of the American mink Mustela vison and its potential impact on the native fauna of Navarino Island, Cape Horn Biosphere Reserve, Chile. Revista Chilena de Historia Natural, 81, 599-613.

Schüttler, E., Klenke, K., McGehee, S., Rozzi, R., \& Jax, K. (2009). Vulnerability of ground-nesting waterbirds to predation by invasive American mink in the Cape Horn Biosphere Reserve, Chile. Biological Conservation, 142, 1450-1460.

Schüttler, E., Ibarra, T., Gruber, B., Rozzi, R., \& Jax, K. (2010). Abundance and habitat preferences of the southernmost population of mink: implications for managing a recent island invasion. Biodiversity and Conservation, 19, 725-743.

Schüttler, E., Rozzi, R., \& Jax, K. (2011). Towards a societal discourse on invasive species management: a case study of public perceptions of mink and beavers in Cape Horn. Journal for Nature Conservation, 19, 175-184.

Sellars, R. W. (1997). Preserving nature in the national parks. A history. New Haven: Yale University Press.

Silander, J., Jr. (2000). Temperate forests: plant species biodiversity and conservation. In S. A. Levin (Ed.), Encyclopedia of Biodiversity (pp. 607-626). New York, USA: Academic Press.

Singh, S. J., Haberl, H., Schmid, M., Mirtl, M., \& Chertow, M. (Eds.). (2013). Long Term Socio-Ecological Research: Studies in Society-Nature Interactions across Spatial and Temporal Scales. New York: Springer.

Touw, M. (1982). Roses in the Middle Ages. Economic Botany, 36, 71-83.

Veblen, T. T., Hill, R. S., \& Read, J. (Eds.). (1996). The ecology and biogeography of Nothofagus forests. New Haven, USA: Yale University Press..

Vidal, O. J., \& Reif, A. (2011). Effect of a tourist- 
ignited wildfire on Nothofagus pumilio forests at Torres del Paine biosphere reserve, Chile (Southern Patagonia). Bosque, 32, 64-76.

Vidal, O. J., San Martín, C., Mardones, S., Bauk, V., \& Vidal, C.F. (2012). The orchids of Torres del Paine Biosphere Reserve: The need for species monitoring and ecotourism planning for biodiversity conservation. Gayana Botanica, 69, 136-146.

Vidal, O. J., Aguayo, M., Niculcar, R., Bahamonde, N., Radic, S., San Martín, C., Kusch, A.,...Félez, J. (2015). Plantas invasoras en el Parque Nacional Torres del Paine (Magallanes, Chile): estado del arte, distribución post-fuego e implicancias en restauración ecológica. Anales del Instituto de la Patagonia, 43, 75-96.

Villarroel, P. (1996). El caso de Puerto Natales - Torres del Paine, XII Región: efecto del turismo en el desarrollo local. Ambiente y Desarrollo, XII(4), 58-64.

Wolf, E. R. (1958). The Virgin of Guadalupe: A Mexican National Symbol. The Journal of American Folklore, 71, 34-35.

Zárraga, C., Massardo, F., \& Rozzi, R. (2006). Hen Larmameuti Hen Maruti Intien: Diccionario Infantil Yagán. Punta Arenas, Chile: Ediciones Universidad de Magallanes. 
